\title{
ON LANGUAGES WHICH ARE BASED ON NON-STANDARD ARITHMETIC ${ }^{1)}$
}

\author{
ABRAHAM ROBINSON
}

1. Introduction. The natural numbers play a part in the formulation of logical syntax inasmuch as they are used to count the symbols in a sentence, or the sentences in a proof, etc. In the present paper, we shall study an infinitary logical calculus which is based on replacing the ordinary natural numbers in the capacity just mentioned, by a non-standard model of arithmetic. (Compare refs. 3, 5, 6 for some other logical calculi of infinitary nature). Thus, our calculus will include formulae of length $n$ for any natural number $n$, finite or infinite, in the chosen non-standard model of arithmetic. Evidently, the study of such formulae can be of interest only if we introduce concepts which are beyond the power of expression of the notions borrowed from the standard case. It turns out that the concept of truth in a model, when defined by means of Skolem functions has this character and involves a curious phenomenon which is analogous to one first pointed out by $\mathrm{H}$. Steinhaus and J. Mycielski for another kind of infinitary language. Thus, while in the standard predicate calculus the negation of a sentence in prenex normal form is reduced to prenex normal form by changing the type of the quantifiers and by shifting the sign of negation, this procedure is not legitimate when truth is defined in this way in our calculus.

The plan of this paper is as follows. In the second section we detail two auxiliary results which are used later. One of them is a generalization, due to Craig (ref. 1), of Beth's theorem on definitions. The second result is Tarski's well known theorem on the non-definability of the set of elementary sentences that are true in arithmetic. The proof of this theorem by means of a non-

Received May 1, 1962.

1) The work on the present paper was carried out in part while the author held a grant from the National Science Foundation (No. G 14006) at the University of California in Berkeley. 
standard model of arithmetic leads up to the main subject of the paper. In the third section, we introduce the non-standard language which forms the main subject of this paper. and discuss its syntactical properties. Finally, sections 4- 6 deal with two types of truth definition for our non-standard language, and their interrelations.

The present paper may thus be regarded as a study of one of the implications of the existence of non-standard models of arithmetic. However, it is not intended as a mere exercise in the use of this concept. In order to appreciate this, notice that in the usual investigations of problems of incompleteness and non-categoricity these phenomena are regarded as properties of certain axicmatic systems, which are detected when the systems in question are investigated from a metamathematical point of view in which arithmetic is supposedly absolute. By introducing non-standard arithmetic already at the stage of the construction of the sentences of the object language, we emphasize the fact that the notion of arithmetic may be relative even at the metamathematical level. However, we do not escape the convenient assumption that at the back of all our investigations there is some system of arithmetic which is absolute.

In the sequel, we shall refer to the Lower predicate calculus without going into the details of the particular version to be used here. In fact, our general arguments apply equally to the various standard versions of the calculus. However, for the sake of definiteness we mention here that we shall envisage a language in which the relation or function symbols do not determine the number of their places. Thus, the same relation symbol may be used, in different connections, to denote both binary and ternary relations. Round brackets are used to enclose the arguments of a relation or of a function. or to enclose a quantifier, while square brackets serve to determine the syntactical grouping. Finally, no quantifier shall contain a variable that is already quantified in its scope.

2. Auxiliary results. Let $L$ be an elementary language (a language of the Lower predicate calculus) whose set of extralogical constants comprises (symbols for) relations, individual constants, and functions. Let $X$ be a sentence in $L$ which contains an $n$-place relation $R, n>0$, and in addition a number of other extralogical constants which constitute a set $S$. Suppose that $S$ is divided into 
two subsets $S_{1}$ and $S_{2}$ such that $S_{1}$ contains at least one $n$-place relation, $m>0$. Let $X^{\prime}$ be the sentence obtained from $X$ by replacing the elements of $S_{2}$, as well as $R$, by distinct extralogical constants of the same kind which were not contained in $X$. Thus, $R$ is replaced by an $n$-place relation $R^{\prime}$ and $S_{2}$ is replaced by a set which will be called $S_{2}^{\prime}$. We may indicate the connection between $X$ and $X^{\prime}$ by writing

$$
X=F\left(S_{1}, S_{2}, R\right), \quad X^{\prime}=F\left(S_{1}, S_{2}^{\prime}, R^{\prime}\right)
$$

The following result, due to W. Craig (ref. 1) is a generalization of Beth's theorem on definitions

\subsection{Theorem. Suppose that}

$$
\vdash X \wedge X^{\prime} \supset\left[\left(\forall x_{1}\right) \cdots\left(\forall x_{i 2}\right)\left[R\left(x_{1}, \ldots, x_{n}\right) \equiv R^{\prime}\left(x_{1}, \ldots, x_{n}\right)\right]\right]
$$

Then there exists a predicate $(w f f) Q\left(x_{1}, \ldots, x_{n}\right)$ whose set of extralogical constants belongs to $S_{1}$ such that

$$
\vdash X \supset\left[\left(\forall x_{1}\right) \cdots\left(\forall x_{n}\right)[R \equiv Q]\right] .
$$

Next, consider an elementary language $N$ whose extralogical constants are the relation of identity, $I(x, y)$ - i.e. $x=y$-, the individual constant 0 , and the functions $\sigma(x, y), \pi(x, y)$ - i.e. $x+y, x y-$, as well as the successor function, which will be denoted by $\phi(x)$. A familiar procedure of arithmetization associates with every atomic symbol, term, wff, and sentence of $N$ a Gödel number such that the set $S$ of Gödel numbers of sentences of $N$ is definable in $N$ i.e. is given by a predicate of $N$ (and is, moreover, recursive). Let $V$ be the set of Gödel numbers of sentences of $N$ which are true for the natural numbers. Then

\subsection{Theorem (Tarski), $V$ is not definable in $N$.}

Proof. Let $x, y$ and $z$ be three arbitrary but definite variables in $N$. Let $P$ be the set of predicates $R(x, y)$ in $N$ which contain no free variables other than $x$ and $y$ and which do not contain $z$. Let $Q$ be the set of predicates $S(x, y)$ which are of the form

$$
R(x, y) \wedge(\forall z)[[(\exists w) I(\sigma(z, w), y)] \supset \sim R(x, z)]
$$

where $R(x, y)$ belongs to $P$. Thus $S(x, y)$ states, in a different notation, that 
$y=\mu z R(x, z)$. The relation between the Gödel number of any element of $P$ and the corresponding element of $Q$ is recursive.

By the length of a formula $R(x, y)$ we mean the number of its atomic symbols, including brackets, connectives, quantifiers, variables, and extralogical constants, separate occurrences being counted separately. The predicate $A(w, t)$, " $w$ is the Gödel number of some formula and $t$ is the length of that formula", is recursive and may be represented as a predicate in $N$. The same applies to the predicate $B(w, t)$, " $w$ is the Gödel number of some formula whose length does not exceed $t$ ". Finally, let $C(u, v, w, t)$ be the predicate " $w$ is the Gödel number of some element $R(x, y)$ of $P$ and $u$ and $v$ are natural numbers, and $t$ is the Gödel number of the formula obtained by substituting the numerals of $u$ and $v$ in the predicate $S(x, y)$ which is given by 2.5." Then $C(u, v, w, t)$ is again recursive and may be taken to be formalized as a predicate in $N$.

Let $M_{0}$ be the system of (non-negative) natural numbers, and suppose, contrary to the assertion of Theorem 2.4 that the set of Gödel numbers of sentences of $N$ which are true in $M_{0}$ is given by a predicate $T(z)$ in $N$. Then the following sentence is true in $M_{0}$,

$$
(\forall u)(\forall s)(\exists v)(\forall w)(\forall t)[B(w, s) \wedge C(u, v, w, t) \supset \sim T(t)]
$$

This sentence states, informally, that for any given natural numbers $u$ and $s$ there exists a natural number $v$ such that for all elements $R(x, y)$ of $P$ whose length does not exceed $s, z=v$ is not the smallest number which satisfies $R(u, z)$. The truth of this sentence in $M_{0}$ follows immediately from the fact that the number of elements of $P$ of length not greater than a given natural number, is finite.

Let $M$ be any non-standard strong model of arithmetic, i.e. a model of $V$ which is a proper extension of $M_{0}$. Let $a$ be an infinite (non-standard) element of $M$. Then the intersection of all elementary submodels of $M$ which include $a$ is known to be a strong non-standard model of arithmetic, which will be denoted by $M_{0}(a)$ (ref. 4). For every element $b$ of $M_{0}(a)$ there exists a predicate $R_{b}(x, y)$ of $P$ such that for the corresponding element $S_{b}(x, y)$ of $Q$ (see 2.5 above), the sentence $S_{b}(\mathbf{a}, \mathbf{b})$ holds in $M_{0}(a)$. (Note that the passage from a number to the corresponding numeral is indicated by bold face print.) 
Now let $c$ be any infinite element of $M_{0}(a)$. Since 2.6 holds in $M_{0}(a)$ it follows that the sentence

$(\exists v)(\forall w)(\forall t)[B(w, \mathbf{c}) \wedge C(\mathbf{a}, v, w, t) \supset \sim T(t)]$

also holds in that structure. Hence, for some element $b$ of $M_{11}(a)$,

$$
(\forall w)(\forall t)[B(w, \mathbf{c}) \wedge C(\mathbf{a}, \mathbf{b}, w, t) \supset \sim T(t)]
$$

Let $R_{b}(x, y)$ be the predicate introduced above. The length of $R_{b}(x, y)$ is a natural number in the ordinary sense, $d$ say, which is smaller than $c$ in $M_{0}(a)$. Let $n$ be the Gödel number of $R_{b}(x, y)$ then $B(\mathbf{n}, \mathbf{c})$ is true in $M_{0}(a)$, and the same applies to the sentence

$$
(\forall t)[B(\mathbf{n}, \mathbf{c}) \wedge C(\mathbf{a}, \mathbf{b}, \mathbf{n}, t) \supset \sim T(t)],
$$

in view of 2.7 , and hence to

$(\forall t)[C(\mathbf{a}, \mathbf{b}, \mathbf{n}, t) \supset \sim T(t)]$

On the other hand, let $m$ be the Gödel number of $S_{b}(\mathbf{a}, \mathbf{b})$ then the sentence $[C(\mathbf{a}, \mathbf{b}, \mathbf{n}, \mathbf{m}) \wedge T(\mathbf{m})]$ holds in $M_{0}(a)$, by the definition of $R_{b}(x, y)$. It follows that

$(\exists t)[C(\mathbf{a}, \mathbf{b}, \mathbf{n}, t) \wedge T(t)]$

holds in $M_{0}(a)$ and this contradicts 2.9. The proof of 2.4 is now complete.

While the above method involves as much arithmetization as any other proof, we were able to avoid the introduction of diagonal arguments by the use of non-standard models. Nevertheless, it may be said that there is a certain relation between our method and the Berry paradox. Essentially, the scope of the method includes Gödel's incompleteness theorem since this is a consequence of Tarski's theorem, given certain ancillary considerations.

Let $W(x)$ be a predicate of $N$ which defines the set of Gödel numbers of sentences of $N$, and let $M$ be any non-standard strong model of arithmetic. Since $W(x)$ is satisfied in $M_{0}$ by an infinite and hence unbounded set of elements, the same is true in relation to $M$. It follows that $W(x)$ holds in $M$ for certain infinite elements. Now there exists a recursive predicate $S(x, y, t)$, " $t$ is the Gödel number of the $y^{t h}$ element of the wff whose Gödel number is $x$ ", which may be taken to be formalized within $N$. The following sentence then holds 
in $M_{0}$ and hence in $M$.

2.11 .

$$
(\forall x)(\forall y)(\forall z)(\exists w)[A(x, y) \wedge Q(z, y) \supset S(x, z, w)] .
$$

In this sentence, $A(x, y)$ signifies " $y$ is the length of the formula whose Gödel number is $x$ ", as before, and $Q(z, y)$ has been introduced to denote the order relation $(\exists v) E(\sigma(z, v), y)$ or, briefly, $z \leq y$. Since there is no uniform bound to the length of the wff of $N$ there exist infinite elements $a$ and $b$ of $M$ such that the sentence $A(\mathbf{a}, \mathbf{b})$ holds in $M$. This sentence states, formally, that $a$ is the Gödel number of a wff of length $b$. This is something more than a purely formal statement since we can actually recover the ordered set of "Gödel numbers of atomic symbols" which constitute the wff defined by $a$. Indeed, 2.11 shows that for any element $c$ of $M$ which does not exceed $b$ there exists an element $d$ of $M$ which is the "Gödel number of the $c^{\text {th }}$ element of $a$ ". If $d$ is finite then it corresponds to one of the original atomic symbols and there is no need to use quotation marks. This will certainly be the case when $c$ is the Gödel number of a connective, or of a bracket, or of a quantifier. However, if $d$ is infinite then we have to regard it as the Gödel number of a new atomic symbol. In order to realize these ideas systematically, it seems advantageous not to limit oneself to the language $N$, which describes arithmetic but to carry out the extersion of the original finitary language within a more general framework. This will be done in the next section.

3. Construction of a non-standard language. Let $U$ be a set of individuals, of a cardinal which is greater than or equal to $2^{\delta_{0}}$, and otherwise arbitrary (unless and until it is defined more closely for a particular purpose). Suppose that distinct symbols have been assigned to all individuals of $U$, to all relations on $U$, and to all functions defined on $U$. Without fear of confusion we denote the corresponding structure again by $U$. In particular, the symbol $I(x, y)$ will be taken to correspond to the relation of identity in $U$. Let $K$ be the set of all sentences of the Lower predicate calculus which are formulated in terms of these extralogical constants and which hold in $U$. Select a countable subset $U_{0}$ of $U$, and let $R_{0}(x)$ be the relation which determines $U_{0}$, i.e. which holds in $U$ precisely for the elements of $U_{0}$. Let $a$ be an individual constant outside the vocabulary of $K$. Consider the set $K^{\prime}=K \cup R_{0}(a) \cup\left\{\sim I\left(a, b_{\nu}\right)\right\}$ where $b$, varies over all elements of $U$. Then a familiar argument shows that $K^{\prime}$ is consistent 
and hence posesses a model, $U^{\prime}$ say. $U^{\prime}$ is a proper extesion of $U$ since it contains $a$ (or an element denoted by $a$ ), and $a$ is different from the elements of $M_{0}$ since it satisfies $\left\{\sim I\left(a, b_{\nu}\right)\right\}$ and from the elements of $U-U_{0}$ since it satisfies $R_{0}(a)$. It is not difficult to see that $I(x, y)$ may be supposed to denote the identity also in $U^{\prime}$.

$U^{\prime}$ is a model of $K$. To every set and relation in $U$ there corresponds a set or relation in $U^{\prime}$ which satisfies the same predicates in the language of $K$. Let $R(x)$ be a predicate of this kind and let $S$ and $S^{\prime}$ be the sets determined by $R(x)$ in $U$ and $U^{\prime}$ respectively. If $S$ is empty then $S^{\prime}$ also is empty since the sentence $(\forall x)[\sim R(x)]$ belongs to $K$. Suppose next that $S$ contains a finite and positive number of elements, $n>0$. Then the sentence $\left(\exists x_{1}\right) \cdots\left(\exists x_{n}\right)(\forall y)$ $\left[R\left(x_{1}\right) \wedge \cdots \wedge R\left(x_{n}\right) \wedge R(y) \supset I\left(x_{1}, y\right) \vee \cdots \vee I\left(x_{n}, y\right)\right]$ holds in $U$ and hence in $U^{\prime}$. Thus $S^{\prime}$ cannot contain more than $n$ different elements. Since $S^{\prime}$ is an extension of $S$ it follows that $S^{\prime}$ coincides with $S$.

Suppose next that $S$ is precisely countable. Then there exists a relation $J(x, y)$ which defines a one-to-one correspondence between $S$ and $U_{0}$ in $U$. Let $U_{0}^{\prime}$ be the set defined by $R_{0}(x)$ in $U^{\prime}$. Then $J(x, y)$ defines a one-to-one correspondence between $S^{\prime}$ and $U_{0}^{\prime}$ in $U^{\prime}$. In particular, for some element $b$ of $S^{\prime}, J(b, a)$ holds in $U^{\prime}$. But $a$ does not belong to $U_{0}$ and so $b$ cannot belong to $S^{\prime}$. Thus, $S^{\prime}$ is a proper extension of $S$.

Finally, if $S$ is an arbitrary infinite subset of $U$ then it contains a countable subset, $S_{0}$ say. If $S^{\prime}$ and $S_{0}^{\prime}$ are the corresponding subsets of $U$ then $S^{\prime} \supseteq S_{0}^{\prime}$ and $S_{0}^{\prime}-S_{0}$ is not empty. But $S_{0}^{\prime} \cap S=S_{0}$ and so $S^{\prime}-S \supseteq S_{0}^{\prime}-S_{0}$, i.e. $S^{\prime}$ is a proper extension of $S$.

Summing up we find that infinite sets are extended on passing from $U$ to $U^{\prime}$ while finite subsets remain unchanged. Now let $M$ be any structure whose domain of individuals, $D$, is a subset of $U$. The relations and functions of $M$ are not in themselves relations and functions of $U$ since they are defined only on $D$. However any function, e.g. $\phi(x, y)$ which is defined on $D$ can be extended - usually in many different ways - to a function $\phi_{1}(x, y)$ which is defined on $U$. A similar statement applies to the relations which are defined on $D$. Suppose then that for every relation and function of $M$ we have selected an extension to the whole of $U$. Let $R(x)$ be the relation which defines $D$ in $U$. For every sentence $X$ which is defined in $M$ we may then construct the 
sentence $X_{1}$ which is obtained from $X$ by replacing every (symbol for a) relation or function in $M$ by the (symbol for) the corresponding extension to $U$ and by relativizing the result with respect to $R(x)$ (for the operation of relativization, see e.g. ref 7 ). Then $X_{1}$ holds in $U$ if and only if $X$ holds in $M$.

Let $H$ be the set of all sentences $X$ which are defined and hold in $M$ and let $H_{1}$ be the set of corresponding sentences $X_{1}$. Then $H_{1} \subseteq K$ and so the sentences of $H_{1}$ hold in $U^{\prime}$. Let $D^{\prime}$ be the subset of $U^{\prime}$ which is determined by $R(x)$ in $U^{\prime}$. By restricting the relations and functions of $H_{1}$ in $U^{\prime}$ to $D^{\prime}$ we then obtain a structure $M^{\prime}$ which is an extension of $M$ and a model of $H$. $M^{\prime}$ is thus an elementary extension of $M$. It is actually independent of the particular choice of the extended relations and functions.

Select a countable subset $D_{0}$ of $U$ and choose functions $\sigma(x, y)$ and $\pi(x, y)$ (i.e. $x+y$ and $x y$ ) on $D_{0}$ in such a way that $D_{0}$ is turned into a standard model of arithmetic $M_{0}$ ("the" natural numbers). Let $M_{0}^{\prime}$ be the corresponding structure in $U^{\prime}$. Then $M_{0}^{\prime}$ also is a strong model of arithmetic and since the underlying domain of individuals $D_{0}^{\prime}$ must be a proper extension of $D_{0}, M_{0}^{\prime}$ is a non-standard strong model of arithmetic.

We now suppose that certain subsets of $U$ are regarded as the constituents of a language $L$ of the first order predicate calculus. That is to say, there are certain disjoint sets of individuals of $U$, of adequate cardinal numbers, which serve as brackets, commas, connectives $(\sim, \wedge$, and $\vee$ ), quantifiers ( $\forall$ and $\exists$ ), variables, individual constants, relations, and functions of $L$. Since they are individuals of $U$, the relations of $L$ will, in general, be distinct from the symbols which denote relations of $U$. Moreover, the relations of $L$ will certainly be different from the relations of $U$ since the latter are sets of $n$-tuples of elements of $U$. Accordingly, we shall call these individuals of $U, L$-relations, and we shall similarly refer to $L$-variables, $L$-connectives, etc.

Going farther, we suppose that the terms and well-ordered formulae (wff) of $L$ also constitute subsets of $U$ (" $L$-terms", $L$-wff"). To regard terms and wff, including sentences, as individuals in a certain domain is entirely in keeping with the axiomatic approach to the syntax of a formal language. Thus, we have in $U$ one-place relations $Q_{v}(x)$, " $x$ is an $L$-variable", $Q_{c}(x)$, " $x$ is an $L$ connective", $Q_{f}(x)$, " $x$ is an $L$-wff", $Q_{s}(x)$, " $x$ is an $L$-sentence", etc. Various connections which exist between these relations are then expressed by sentences 
of $K$, for example

$$
(\forall x)\left[Q_{s}(x) \supset Q_{f}(x)\right] .
$$

Again, there exists a relation $S(x, y, z)$ in $U$ which states that $x$ is an $L$-wff and $y$ is a natural number (an element of $D_{0}$ ) and $z$ is the $y^{\text {th }}$ atomic symbol ( $L$-bracket, $L$-relation, $L$-variable, etc.) in $X$. Then the following sentences belong to $K$.

$(\forall z)(\forall w)[S(x, y, z) \wedge S(x, y, w) \supset I(z, w)]$,

$$
(\forall x)(\forall y)[[(\forall u)(\forall v)[S(x, u, v) \equiv S(y, u, v)]] \supset I(x, y)] \text {. }
$$

The second sentence involves the tacit assumption, which is usually taken for granted, that a sentence is determined completely by its sequence of atomic symbols. We note that any discussion of the question how to relate different occurrences of the same symbol is entirely redundant in the present approach.

Passing to $U^{\prime}$, we see that the relations which in $U$ define the various sets of $L$-symbols and $L$-formulae, define corresponding sets in $U^{\prime}$. If the original sets are finite, as is the case for the connectives and for the quantifiers, then they remain unchanged on passing to $U^{\prime}$. If the original set is infinite, as is the case for the sentences, then the corresponding set in $U^{\prime}$ is a proper extension of it. The extended language will be denoted by $L^{\prime}$ and, accordingly, we shall refer to its variables, connectives, and so on, as $L^{\prime}$-variables, $L^{\prime}$-connectives, etc. Now let $F$ and $F^{\prime}$ be the sets of $L$-wff and of $L^{\prime}$-wff, respectively. Then $F \subseteq U$, $F^{\prime} \subseteq U^{\prime}$ and $F$ is a proper subset of $F^{\prime}$. Every element $a$ of $F^{\prime}$ determines a certain ordered set of atomic symbols in the sense that for every element $n$ of $D^{\prime}$ (i.e. for every natural number in $U^{\prime}$ ) which does not exceed a particular $m \in D^{\prime}$ - the length of $a$-there is a unique $L^{\prime}$-atomic symbol $b$ such that $S(a, n, b)$ holds in $U^{\prime}$, where $S(x, y, z)$ is the relation introduced above (" $b$ is the $n^{\text {th }}$ atomic symbol of $\left.a^{\prime \prime}\right)$. We know that such $m$ and $b$ exist because this fact is expressed by an elementary sentence which holds in $U$.

The set of $L^{\prime}$-wff and more particularly, of $L^{\prime}$-sentences, is quite varied. Thus, for every non-standard natural number $l$ in $U^{\prime}$ there exists an $L^{\prime}$-sentence whose length exceeds $l$. That this is so can be deduced immediately from the existence of sentences of unbounded length in $U$. Again, if $L$ includes relations of $n$ places for all natural numbers $n$, then $L^{\prime}$ will include relations whose number of places, $n$, is infinite, more precisely $n$ is an infinite element of $M_{0}^{\prime}$. 
On the other hand, we cannot assign the elements of an infinitely long wff in the present sense arbitrarily even in cases where this would appear to yield a formula which is intuitively meaningful, as will be shown presently.

We recall that the order type of any non-standard model of arithmetic can be expressed in the form $\omega+\left({ }^{*} \omega+\omega\right) \theta$ where $\theta$ is some dense order type without first or last element (compare ref. 2). It follows that if $m$ is an infinite element of $M^{\prime}$ then the ordered set of elements of $M^{\prime}$ which are smaller than or equal to $m$ is of order type $\omega+\left({ }^{*} \omega+\omega\right) \theta^{\prime}+{ }^{*} \omega$ where $\theta^{\prime}$ is again dense without first or last element. Accordingly the ordered sequence of any $L^{\prime}$-wff which is of infinite length possesses an order type of this kind.

To continue, we require some simple facts concerning finite partial sequences. By a finite partial sequence $\sigma$ we mean any function whose domain is a finite set of natural numbers and whose range is included in a given set, $G$. By the length of $\sigma$ we mean the greatest element in the domain of $\sigma$ plus 1 . The length of the empty sequence (sequence with empty domain) shall be zero, by definition.

The number of non-empty finite partial sequences with range in $U$ is equal to the cardinal of $U$. Accordingly these sequences can be indexed in $U$ in the following sense. There exists a subset $J$ of $U$ and a three place relation $N(x, y, z)$ on $U$ such that $x$ is an element of $J$ and $y$ is a natural number (an element of $M_{0}$ ) and such that the following conditions are satisfied

$$
(\forall x)(\forall y)(\forall z)(\forall w)[N(x, y, z) \wedge N(x, y, w) \supset I(z, w)] .
$$

$$
(\forall x)(\exists y)(\forall z)(\forall w)[N(x, z, w) \supset Q(z, y)] \text {. }
$$

$$
(\forall x)(\forall y)[[(\forall z)(\forall w)[N(x, z, w) \equiv N(y, z, w)]] \supset I(x, y)] \text {. }
$$

Of these, 3.3 states that, for given $x, N(x, y, z)$ defines $z$ as a function of $y, 3.4$ affirms that the domain of any such function is bounded by a natural number $y$ (where $Q(z, y)$ denotes the relation $z \leq y$ between natural numbers, as before) and 3.5 ensures that no two different elements of $J$ define the same function.

Morever, we suppose that as $x$ varies over the elements of $J$ all finite partial sequences with values in $U$ occur as functions defined by $N(x, y, z)$. It is evident that this condition can be satisfied (in many ways) by a suitable choice of $J$ and $N$, but it does not correspond to any sentence in $K$.

Suppose that $J$ is determined by a relation $Q_{c}(x)$ in $U$. Let $G$ be any 
subset of $U$ then we may obtain all finite partial sequences with values in $G$ by restricting $J$ to a suitable subset, $J_{G}$ say. Let $Q_{\theta}(x)$ be the relation which determines $J_{G}$ then we may define a corresponding three place relation $N_{G}(x, y, z)$ by

$$
N_{G}(x, y, z)=Q_{G}(x) \wedge N(x, y, z) .
$$

We note that as a consequence of our stated assumptions the sentence

$$
(\forall x)\left[[(\exists y)(\exists z) N(x, y, z)] \equiv Q_{U}(x)\right]
$$

belongs to $K$. (We have excluded the empty sequence from the indexing, otherwise the equivalence in 3.7 would have to be replaced by an implication.) Accodingly, $J$ is determined completely by $N(x, y, z)$. Similarly, for any $G \subseteq U$, $J_{G}$ is determined by $N_{G}$.

Passing to $U^{\prime}$, we find that $Q_{U}(x)$ determines a subset $J^{\prime}$ of $U^{\prime}$ which contains $J$ as a proper subset. Any element $a$ of $J$ then defines a partial sequence with domain in $M_{0}^{\prime}$ by means of the predicate $N(a, y, z)$. Indeed, this predicate defines $z$ as a function of $y$ in view of 3.3, such that the arguments are in $M_{0}^{\prime}$ by virtue of an earlier condition. Moreover, in view of 3.4 , the domain of the sequence is bounded by some element of $M_{0}^{\prime}$. Any function which is defined in this way by some $N(a, y, z)$ will be called a pseudo-finite partial sequence. Notice that the set of pseudo-finite partial sequences in $U^{\prime}$ does not depend on the particular choice of $N(x, y, z)$ (which implies a definite choice for $J$ ). Indeed, suppose that we have chosen two different relations on $U, N_{1}(x, y, z)$ and $N_{2}(x, y, z)$, with corresponding index sets $J_{1}$ and $J_{2}$ in such a way that all the conditions laid down for $N$ and $J$ are satisfied by $N_{1}$ and $J_{1}$ and by $N_{2}$ and $J_{2}$, respectively. Let $Q_{1}(x)$ and $Q_{2}(x)$ determine the sets $J_{1}$ and $J_{2}$, respectively. Then if $a$ belongs to $J_{1}, N_{1}(a, y, z)$ defines a non-empty finite partial sequence in $U$. But this sequence must occur also for some value of the first argument in $N_{2}(x, y, t)$. Accordingly, the sentences

$$
(\forall x)\left[Q_{1}(x) \supset\left[(\exists w)\left[(\forall y)(\forall z)\left[N_{1}(x, y, z) \equiv N_{2}(w, y, z)\right]\right]\right]\right.
$$

and

$$
(\forall x)\left[Q_{2}(x) \supset\left[(\exists w)\left[(\forall y)(\forall z)\left[N_{2}(x, y, z) \equiv N_{1}(w, y, z)\right]\right]\right]\right]
$$

hold in $U$ and hence belong to $K$. Thus, the two sentences hold also in $U^{\prime}$ and 
this shows that the sets of pseudo-finite partial sequences defined by $N_{1}$ and $N_{2}$ coincide. The set of non-empty finite partial sequences in $U$ will be denoted by $\Sigma$ and the set of non-empty pseudo-finite partial sequences in $U^{\prime}$ by $\Sigma^{\prime}$. We shall imagine that the index set $J$ has been fixed once and for all. A sequence will be said to be total if its domain contains with every number also all smaller numbers. Such sequences form subsets $\Sigma_{0}$ and $\Sigma_{0}^{\prime}$ of $\Sigma$ and $\Sigma^{\prime}$ respectively.

Now let $T$ be the set of $L$-sentences, $T \subseteq U$, and let $\Sigma_{T}$ be the subset of $\Sigma_{0}$ whose elements take values in $T$, i.e. which are finite partial sequences of $L$-sentences. Let $J_{T} \subseteq J$ be the corresponding index set. Then the rule which assigns to every non-empty finite total sequence of $L$-sentences, $\sigma=\left(p_{0}, \ldots, p_{n}\right)$ the $L$-sentence

3.8.

$$
q=\left[p_{0} \wedge\left[p_{1} \wedge\left[p_{2} \wedge \cdots \wedge p_{n}\right] \cdots\right]\right.
$$

defines a function $\psi$ on $\Sigma_{T}$, where $q=\psi(t)$ if $t$ is the index of $\sigma$ in $J . \quad \psi$ can be represented by a relation $Q \psi(x, y)$ which holds if $x$ is the index of the sequence $\sigma=\left(p_{0}, \ldots, p_{n}\right)$ and $y$ is given by 3.8. Passing to $U^{\prime}$ we see that $\boldsymbol{Q} \psi(x, y)$ assigns a sentence also to every non-empty pseudo-finite total sequence.

We note that if in 3.8 we count the symbols from left to right, beginning with 0 , then the places with index $\equiv 0(3)$ are filled with left brackets, those with index $\equiv 1(3)$ by sentences, and those with index $\equiv 2(3)$ by the connective of conjunction, up to the place with index $3 n-1$. The place with index $3 n$ is filled by the last sentence, $p_{n}$. This is followed by $n$ right brackets. Thus, the length of $q$ is $4 n+1$.

Let $n$ be an infinite natural number in $M_{0}^{\prime}$ and let $a$ and $b$ be two distinct $L$-sentences. It would then appear to be intuitively possible to define a particular $L^{\prime}$-sentence $r$ of length $4 . n+1$ which is a repeated conjunction of the sentences $a$ and $b$ simply by substituting in $s$ and $q$ (see 3.8) the sentence $a$ for $p_{i}, i$ finite and the sentence $b$ for $p_{i}, i$ infinite, $i \leq n$. This is indeed a well-defined ordered set of atomic symbols, of length $4 n+1$ as we can see from the detailed description given in the preceding paragraph. However, it is not an $L^{\prime}$-sentence. For if $r$ were an $L^{\prime}$-sentence then the sentences which fill the places with index $3 k+1$ in $r, 0 \leq k \leq n-1$ would constitute a pseudo-finite total sequence $\rho$ of length $n$ such that the elements of the sequence are equal to $a$ for all finite 
subscripts and to $b$ for all infinite subscripts. Such a sequence $\rho$ cannot belong to $\Sigma_{0}^{\prime}$. For, using an argument which can easily be formalized, $\rho$ satisfies the condition that its $0^{t h}$ element is $a$ and that whenever an element of $\rho$ equals $a$ the next element, if any, is also equal to $a$. Hence by the axiom of induction which holds in $U^{\prime}$, if $\rho \in \Sigma_{0}^{\prime}$ then all elements of $\rho$ are equal to $a$, contrary to construction. Thus, there is no $L^{\prime}$-sentence of the kind described above.

The deductive calculus of the language $L^{\prime}$ will be discussed only briefly. We take for granted the deductive calculus of the language $L$. More particularly, we take it that there exists a subset $T_{0}$ of the set of sentences $T$ which is constituted by the $L$-axioms. An $L$-proof is a non-empty finite total sequence of $L$-sentences such that every element of the sequence is either an $L$-axiom of it is deducible from one or two earlier elements of the sequence as an immediate consequence in accordance with the usual rules of the lower predicate calculus. Thus, the proofs constitute a subset $\Sigma_{P}$ of $\Sigma_{T}$. Let $J_{P}$ be the subset of $J$ whose elements induce the elements of $\Sigma_{P}$ and let $Q_{P}(x)$ be the relation on $U$ which determines $J_{P}$.

An $L$-sentence is provable if it is an element of some $L$-proof. Thus, " $z$ is provable" corresponds to the predicate $(\exists x)(\exists y)\left[Q_{P}(x) \wedge N(x, y, z)\right]$. The set of provable sentences in $U$ will be denoted by $T_{P}$.

More generally, if $A$ is a set of $L$-sentences in $U$, which is given by a relation $Q_{A}(x)$, then we define an $A$-proof as a non-empty finite total sequence of $L$-sentences such that every element of the sequence is either an $L$-axiom or an element of $A$ or it is deducible from one or two earlier elements of the sequence as an immediate consequence. For given $A$, the $A$-proofs constitute a subset $\Sigma_{A}$ of $\Sigma_{T}$. Let $J_{A}$ be the subset of $J$ whose elements index the elements of $\Sigma_{A}$ and let $Q_{A}(x)$ be the relation on $U$ which determines $J_{A}$. An $L$-sentence is $A$-provable if it is an element of some $A$-proof. Thus, " $z$ is $A$-provable" is given by $(\exists x)(\exists y)\left[Q_{A}(x) \wedge N(x, y, z)\right]$. The set of $A$-provable sentences will be denoted by $T_{A}$.

The predicate $Q_{P}(x)$ defines in $U^{\prime}$ a set of $L^{\prime}$-sentences, $T_{P}^{\prime}$ which is an extension of $T_{P}$. The elements of $T_{P}^{\prime}$ will be said to be $L^{\prime}$-provable. An $L$-sentence which is not $L$ provable is not $L^{\prime}$-provable either. On the other hand if $T_{0}^{\prime}$ is the set of $L^{\prime}$-axioms (corresponding to the set $T_{0}$ in $U$ ) then it can be shown that not all sentences which are $L^{\prime}$-provable are deducible from 
$T_{0}^{\prime}$ by the rules of the lower predicate calculus. Indeed, since for any natural number $n$ there exist $L$-provable sentences which cannot be proved in less than $n$ steps, or more precisely which do not occur in proofs of length $<n$, it follows that there exist $L$-provable sentences which do not occur in any proof of finite length.

More generally, let $A^{\prime}$ be any set of $L^{\prime}$-sentences. Then we define the set $T_{A}^{\prime}$ of $L^{\prime}$-sentences which are $A^{\prime}$-provable in the following way. Among the subsets of $B^{\prime}$ of $A^{\prime}$ there are some which are defined by relations that occur already in $U$, i.e. which correspond to sets of $L$-sentences $B$ in $U$. Among these subsets $B^{\prime}$ of $A^{\prime}$ there are, for example, all finite subsets of $A^{\prime}$. For any $B$ which corresponds to such a $B^{\prime}$ there exists a relation $R_{B}(z)$ which determines the $B$-provable sentences in $U . R_{B}(z)$ determines a set $T_{B}^{\prime}$ in $v^{\prime}$. Then $T_{A^{\prime}}^{\prime}$ is by definition the union of all such sets. In particular, if the original $A^{\prime}$ is itself a set which corresponds to a set of $L$-sentences, $A$, then $T_{A^{\prime}}^{\prime}$ corresponds, in $U^{\prime}$ to the set of $A$-provable sentences in $U$.

4. Semantics of a non-standard language. In the remainder of this paper, we shall be concerned with the semantics of the non-standard language $L^{\prime}$ in an extension $U^{\prime}$ of $U$. For this purpose, we first have to discuss the notion of a truth definition in the standard language $L$ within the framework of the set $U$.

Let $M$ be a structure whose domain of individuals, $D$, is a subset of $U$, with a set of relations $P(M)$ and a set of functions $F(M)$. Let $R(x)$ be the relation which determines $D$ in $U$. As discussed in detail at the beginning of the preceding section, the elements of $P(M)$ and of $F(M)$ are not, in general, defined on $U$, but the sentences about $M$ which involve the elements of $P(M)$ and $F(M)$ can be related to sentences about $U$ by means of the operation of relativization with respect to $R(x)$.

We now wish to use the language $L$ in order to describe the structure $M$. For this purpose, we first introduce correspondences between the individuals of $D$ and some $L$-individuals; between the relations of $P(M)$ and some $L$ relations; and between the functions of $F(M)$ and some $L$-functions. It will be assumed that a sufficient number of $L$-symbols is available for this purpose.

Let $Q_{i c}(x)$ be the relation which determines the set of $L$-individuals in $U$, and suppose that a correspondence between the elements of $D$ and a set of 
$L$-individuals is provided by a relation $C(x, y)$ on $U$ which satisfies the following conditions.

4. 1 .

$$
(\forall x)(\forall y)\left[C(x, y) \supset R(x) \wedge Q_{i c}(y)\right]
$$

$$
(\forall x)(\forall y)(\forall z)[C(x, y) \wedge C(x, z) \supset I(y, z)] \text {, }
$$

$$
(\forall x)(\forall y)(\forall z)[C(y, x) \wedge C(z, x) \supset I(y, z)],
$$

4.4 .

$$
(\forall x)(\exists y)[R(x) \supset C(x, y)] \text {. }
$$

The sentences $4.1-4.4$ state, briefly, that $C(x, y)$ establishes a one-to-one correspondence between $D$ and a set of $L$-individuals. The set of $L$-individuals which corresponds to individuals of $M$ will be denoted by $G$. It is given by the predicate $(\exists x) C(x, y)$.

The correspondence between the elements of $R(M)$ and a set of $L$-relations is expressed in a way which is rather different. We suppose that for every element $R\left(x_{1}, \ldots, x_{n}\right)$ of $P(M), n \geq 0$, we are given an $L$-relation $r$ and an $(n+1)$-place relation on $U, S_{R}\left(y_{0}, y_{1}, \ldots, y_{n}\right)$ such that different $L$-relations correspond to different elements of $R(M)$ and such that the sentences

$$
\begin{aligned}
& \text { 4.5. }\left(\forall x_{1}\right) \cdots\left(\forall x_{n}\right)\left(\forall y_{1}\right) \cdots\left(\forall y_{n}\right)\left[C\left(x_{1}, y_{1}\right) \wedge \cdots \wedge C\left(x_{n}, y_{n}\right) \supset\right. \\
& \left.\left[R\left(x_{1}, \ldots, x_{n}\right) \equiv S_{R}\left(r, y_{1}, \ldots, y_{n}\right)\right]\right]
\end{aligned}
$$

all belong to $K$. Let $G_{P}$ be the set of $L$-relations which are employed in this way.

Similarly, we suppose that there is a one-to-one correspondence between the elements of $F(M)$ and a certain subset $G_{F}$ of the set of $L$-functions such that for every function $\phi\left(x_{1}, \ldots, x_{n}\right) \in F(M)$ and the corresponding $g \in G_{F}$ there exists an $(n+2)$-place relation $S_{\phi}\left(y_{0}, y_{1}, \ldots, y_{n+1}\right)$ on $U$ for which

$$
\begin{array}{r}
\text { 4.6. } \quad\left(\forall x_{1}\right) \cdots\left(\forall x_{n}\right)\left(\forall x_{n+1}\right)\left(\forall y_{1}\right) \cdots\left(\forall y_{n}\right)\left(\forall y_{n+1}\right)\left[C\left(x_{1}, y_{1}\right) \wedge \cdots \wedge\right. \\
C\left(x_{n}, y_{n}\right) \wedge C\left(x_{n+1}, y_{n+1}\right) \supset\left[I\left(\phi\left(x_{1}, \ldots, x_{n}\right), x_{n+1}\right)\right. \\
\left.\left.\equiv S_{\phi}\left(g, y_{1}, \ldots, y_{n}, y_{n+1}\right)\right]\right]
\end{array}
$$

belongs to $K$.

We do not exclude the possibility that the set of $L$-individuals, $G$, coincides with the set of individuals of $M, D$, and that at the same time, the relation $C(x, y)$ coincides with the restriction of the relation of identity $I(x, y)$ to $D$. Even if this is not the case from the outset we may, if we wish, define a structure $M^{*}$ isomorphic to $M$ which possesses that property. Thus, if $R$ is a 
relation of $M$ then we choose the corresponding relation of $M^{*}$ on the set of individuals $G$ as the relation which satisfies

4.7. $\left(\forall x_{1}\right) \cdots\left(\forall x_{n}\right)\left(\forall y_{1}\right) \cdots\left(\forall y_{n}\right)\left[C\left(x_{1}, y_{1}\right) \wedge \cdots \wedge C\left(x_{n}, y_{n}\right) \equiv\right.$

$$
\left.\left[R\left(x_{1}, \ldots, x_{n}\right) \supset R^{*}\left(y_{1}, \ldots, y_{n}\right)\right]\right] .
$$

In other words, $R^{*}\left(y_{1}, \ldots, y_{n}\right)$ is coextensive with the predicate $S_{R}\left(r, y_{1}, \ldots, y_{n}\right)$. The corresponding functions of $M^{*}$ may be defined in a similar way.

We also observe that while the correspondences between the elements of $D, R(M)$, and $F(M)$, and $G, G_{R}$ and $G_{F}$, respectively, have to be fixed in order to make the semantical interpretation of an $L$-sentence in $M$ definite, the particular choice of $S_{R}$ and $S_{\phi}$ is irrelevant.

Let $G_{T}$ be the set of all $L$-sentences whose atomic symbols include no extralogical constants other than those belonging to $G \cup G_{R} \cup G_{F}$. Since we are at the moment dealing with the language $L$ which is based on standard arithmetic it is not difficult to reformulate the usual procedure for the determination of the truth or falsehood of a sentence of $G_{T}$ with respect to the structure $M$ within the present framework.

Employing the usual terminology, we call a sentence atomic if it does not involve any connectives or quantifiers and hence does not involve any variables. Disregarding the brackets and commas, an atomic sentence consists of a relation which is followed by a sequence of terms. The terms themselves are not atomic symbols but are given by sequences of functions and of individuals combined according to certain definite rules. Let the atomic $L$-sentence $a \in G_{T}$ be indicated by the expression $r\left(t_{1}, \ldots, t_{n}\right)$ where $r$ is an $L$-relation and $t_{1}, \ldots, t_{n}$ are $L$-terms. By virtue of 4.6 every term $t_{i}$ corresponds to an individual $b_{i}$ of $D$ and, again by means of the correspondence $r$ itself corresponds to a relation $R$. We now ask whether $R\left(b_{1}, \ldots, b_{n}\right)$ holds in $M$. As it stands this question does not refer directly to the structure $U$, but as explained earlier all sentences about $M$ can be reduced to sentences about $U$. Thus, finally, we say that $a$ holds in $M$, or is true in $M$ if and only if $R\left(b_{1}, \ldots, b_{n}\right)$ holds in $M$. The truth values of all other sentences of $G_{T}$ in $M$ are now determined by means of the conditions that a negation is true in $M$ iff the original $L$-sentence is not true in $M$, a disjunction is true in $M$ iff at least one of its disjuncts is true, etc. together with the usual conditions for the quanti- 
fications. Note that the connection be`ween $L$-sentence and its negation, or of two $L$-sentences and their disjunction is given by two and three place relations defined on $U$, e.g. $N(x, y)$ " $x, y$ are $L$-sentences and $y$ is the negation of $x$ ". The relation between $x$ and $y$ can be analyzed further by means of the strings of atomic symbols which define any $\bar{L}$-sentence but this is not necessary for our present purpose. Let the set of all $L$-sentences which hold in $M$ according to this definition be denoted by $G_{M}$ and let $Q_{T}(x)$ and $Q_{u}(x)$ be the relations of $U$ which determine $G_{T}$ and $G_{M}$, respectively.

On passing to $U^{\prime}$, the structure $M$ is transformed into a structure $M^{\prime}$ which is an extensicn of $M$ and that all sentences (in the ordinary sense) which are defined and hold in $M$, hold also in $M^{\prime}$ (i.e. $M^{\prime}$ is an elementary extension of $M$ ). We now define that the set of $L^{\prime}$-sentences which hold in $M^{\prime}$ ascording to the internal truth definition (or briefly, internally) is the set $G_{M}^{\prime}$ which is determined by the relation $Q_{M}(x)$ in $U^{\prime}$. Among the elements of $G_{M}^{\prime}$ are the $L$-sentences of $G_{M}$ which correspond in a natural way to the sentences (in the ordinary sense) that hold in $M$. Beyond that, $G_{M}^{\prime}$ contains also a wide variety of additional sentences including sentences of infinite length. All the ordinary rules of semantics which were mentioned above still hold in the present case. Thus, the negation of an $L^{\prime}$-sentence holds in $M^{\prime}$ iff the sentence does not hold in $M^{\prime}$, a conjunction holds in $M^{\prime}$ iff both conjuncts hold in that structure, a disjunction holds in $M^{\prime}$ iff at least one of the disjuncts holds in $M^{\prime}$. If $q(x)$ is an $L^{\prime}$-wff with a single free variable and with extralogical constants in $C^{\prime}$ $C_{P}^{\prime} C_{F}^{\prime}$ then $(\exists x) q(x)$ (or, more precisely, the $L^{\prime}$-sentence which is obtained from $q(x)$ by existential quantification) holds in $M^{\prime}$ iff $q(a)$ holds in $M^{\prime}$ for at least one $a \in b^{\prime}$ and $(\forall x) q(x)$ holds in $M^{\prime}$ iff $q(a)$ holds in $M^{\prime}$ for all $a \in b^{\prime}$.

Now let $G_{Q}$ be the set of $L$-predicates ( $L$-wff with at least one free variable) whose extralogical constants belong to $G \cup G_{P} \cup G_{F}$. Such an $L$-predicate is said to be defined in $M$. Let $\left(x_{0}, x_{1}, \ldots, x_{n}\right)$ be the finite total sequence of variables of an element $q$ of $G_{Q}$, taken in their order of first occurrence from left to right. Let $\Sigma_{0}(M)$ be the set of all finite total sequences in $U$ whose elements belong to $M$ and let $H_{M}$ be the subset of $J$ which indexes the elements of $\Sigma_{0}(M)$. Let $R_{Q}(x)$ and $H(x)$ be the relations which determine the sets $G_{Q}$ and $H_{M}$ in $U$.

Then the set $G_{Q}^{\prime}$ which is determined by $R_{Q}(x)$ in $U^{\prime}$ is precisely the set 
of all $L^{\prime}$-predicates which are defined in $M^{\prime}$, i.e. whose set of extralogical constants belongs to $G^{\prime} \cup G_{P}^{\prime} \cup G_{F}^{\prime}$. Similarly, the set $H_{\mu^{\prime}}^{\prime}$ defined by $H(x)$ in $U^{\prime}$ indexes the set $\Sigma_{0}^{\prime}\left(M^{\prime}\right)$ of all pseudo-finite total sequences in $U^{\prime}$ whose elements belong to $M^{\prime}$.

The condition " $x$ is an $L$-predicate which is defined in $M$ and $y$ is the index of an element $\sigma$ of $\Sigma_{0}(M)$ whose length equals the number of free variables of $x$, and $z$ is the $L$-sentence resulting from the substitution of the elements of $\sigma$ in their given order for the free variables of $x$ " can be expressed by a relation $W(x, y, z)$ in $U$. Then $W(x, y, z)$ has a corresponding meaning in $U^{\prime}$, where the number of free variables in a given predicate may now be infinite. In either $U$ or $U^{\prime}$, if $x$ is an $L$-( or $L^{\prime}$-) predicate which is defined in $M\left(M^{\prime}\right)$ and $y$ is the index of an element $\sigma$ of $\Sigma_{0}(M)$ (or $\Sigma_{0}^{\prime}\left(M^{\prime}\right)$ ) then the corresponding $z$ is an $L$-(or $L^{\prime}$ ) sentence which is defined in $M$ (or $M^{\prime}$ ). In particular, if $q$ is an element of $G_{Q}^{\prime}$ and the number of variables in $q$ is infinite, then these variables constitute a pseudo-finite total sequence $\xi=\left(x_{3}, x_{1}, \ldots, x_{n}\right)$. The substitution of an element $\sigma=\left(a_{0}, a_{1}, \ldots, a_{n}\right)$ of $\Sigma_{0}^{\prime}\left(M^{\prime}\right)$ for $\xi$ yields an $L^{\prime}$-sentence which is defined in $M^{\prime}$. Conversely, it is not difficult to see that if $\sigma$ is an ordered set of elements of $M^{\prime}$ whose order type is the same as that of $\xi$, and if the substitution of the elements of $\sigma$ for the corresponding elements of $\xi$ in $q$ yields an $L^{\prime}$-sentence then $\sigma$ belongs to $\Sigma_{0}^{\prime}\left(M^{\prime}\right)$. For given $n$, the elements of $\Sigma_{0}^{\prime}\left(M^{\prime}\right)$ of length $n+1$ are divided into two classes according as the result of the substitution does or does not hold in $M^{\prime}$. Accordingly, $q$ defines a kind of infinitary pseudo-relation in $M^{\prime}$ which is defined for some but not all ordered sets of elements of $M^{\prime}$ which have the order type of $\xi$.

5. Skolem operators and the external truth definition. To continue we require some auxiliary considerations which yield a modified version of Henkin's notion of generalized quantifiers (ref. 3). We shall be concerned only with linearly ordered quantification.

Let $S$ be an ordered set which is partitioned into two subsets, $S_{1}$ and $S_{2}$. (Thus, $S_{1} \cup S_{2}=S, S_{1} \cap S_{2}=\emptyset$ ). Let $\Phi_{1}$ and $\Phi_{2}$ be two sets of functions which are defined in $S_{1}$ and $S_{2}$ respectively and which take values in a given set $A$. By a Skolem operator we mean a function $\psi$ whose domain is $\Phi_{1}$ and whose values belong to $\Phi_{2}$ (i.e. a mapping from the functions of $\Phi_{1}$ to functions of $\Phi_{2}$ ) 
in such a way that if $g=\psi(f)$ and $k=\psi(h)$ and $f\left(s_{1}\right)=h\left(s_{1}\right)$ for all elements $s_{1}$ of $S_{1}$ which precede any particular $s_{2} \in S_{2}$ in the given ordering of $S$, then $g\left(s_{2}\right)=k\left(s_{2}\right)$.

Thus, if $X$ is a sentence in prenex normal form in the Lower predicate calculus,

$$
X=q_{0} q_{\mathrm{i}} \cdots q_{n-1} Z\left(x_{0}, x_{1}, \ldots, x_{n-1}\right)
$$

where, for $i=0, \ldots, n-1$ the quantifier $q_{i}$ quantifies the variable $x_{i}$, and where $Z$ does not contain any quantifiers, let $S$ be the ordered set $(0,1, \ldots$, $n-1$ ), and let $S_{1}$ and $S_{2}$ be the subsets of $S$ whose elements are, respectively, the subscripts of the universal and of the existential quantifiers of $X$. Suppose that $X$ holds in a structure $M$ with domain of individuals $A$. Then, corresponding to every existential quantifier in $X$, we may define a Skolem (or Herbrand) function of the preceding universally quantified variables in such a way that the introduction of these functions into $Z$ yields a true sentence for all values of the remaining arguments of $Z$. For example, if $X=\left(\forall x_{0}\right)\left(\exists x_{i}\right)$ $\left(\forall x_{2}\right)\left(\exists x_{3}\right) Z\left(x_{0}, x_{1}, x_{2}, x_{3}\right)$ holds in $M$ then there are Skolem function $\phi\left(x_{0}\right)$, $\phi_{3}\left(x_{0}, x_{2}\right)$, such that $Z\left(x_{0}, \phi_{1}\left(x_{0}\right), x_{2}, \phi_{3}\left(x_{0}, x_{2}\right)\right)$ holds in $M$ for all values of the arguments $x_{0}, x_{2}$ in $A$. Defining $S=(0,1,2,3), S_{1}=(0,2), S_{2}=(1,3), \Phi_{1}$ as the set of all functions on 0,2 and taking values in $A, \Phi_{2}$ as the set of all functions on 1,3 and taking values in $A$, we may regard the mapping $\left(x_{0}, x_{2}\right)$ $\rightarrow\left(\phi_{1}\left(x_{0}\right), \phi_{3}\left(x_{0}, x_{2}\right)\right)$ as a Skolem operator. The same is true for general $X$, as above. Conversely (at first disregarding the question whether the substitution of the Skolem functions makes the matrix true universally) any Skolem operator from the set of functions of elements of $A$ defined on $S_{1}$ into the set of functions of elements of $A$ defined on $S_{2}$ yields a set of Skolem functions. Accordingly, we may say that a sentence $X$ as above holds in a structure $M$ if and only if there exists a Skolem operator which satisfies the condition just described.

We now consider these notions in connection with the language $L$. Let $J$ be an index set for all partial finite sequences in $U$ as before, except that in the present section we shall for convenience suppose that $J$ includes an index for the empty sequence. Let $a_{1}$ and $a_{2}$ be indexes for two disjoint finite total sequences of natural numbers (elements of $\left.M_{0}\right) \sigma_{1}$ and $\sigma_{2}$. The number of such pairs, $\left(\sigma_{1}, \sigma_{2}\right)$, is countable. Let $\Phi_{i}$ be the set of all finite partial sequences 
whose domain is $\sigma_{i}$ and whose values are $L$-individuals, $i=1,2$. Then the cardinal of both $\Phi_{1}$ and $\Phi_{2}$ is not greater than (and, except for empty $\sigma_{i}$ is equal to) the cardinal of the set of all $L$-individuals, $\lambda$ say. We shall now suppose that the cardinal of $U$ is not less than $2^{\lambda}$. Since the number of Skolem operators from $\Phi_{1}$ to $\Phi_{2}$ does not exceed $2^{\lambda}$, it follows that there exists a set $J_{\psi}$ in $U$ which indexes the Skolem operators. Various relations may now be associated with $J_{\psi}$. For example, there exists a relation $A_{\psi}(x, y, z, u, v, w)$ which signifies " $x$ and $y$ are indexes of disjoint finite total sequences of natural numbers as above, $\sigma_{1}$ and $\sigma_{2}$ say, $z$ is the index of a Skolem operator as defined, from sequences with domain $\sigma_{1}$ and values in the set of $L$-individuals to sequences with domain $\sigma_{2}$ and values in the set of $L$-individuals, $u$ is the index of a particular partial sequence of $L$-individuals with domain $\sigma_{1}, v$ is a natural number which is an element of $\sigma_{2}$, and $w$ is the value of $\phi(t)$ for the argument $v . "$

Let $X$ be any sentence. By a prefix of $X$ we mean any sequence of quantifiers $Q$ such that $X=Q Z$ where $Z$ may contain further quantifiers. We may consider the same notion also for $L$-sentences. Accordingly, there exists a relation $Q_{p r}(t, x, y)$ which has the following significance. " $t$ is an $L$-sentence which possesses a prefix $\pi$ such that $x$ is the index of the sequence of (finite) ordinal numbers which correspond to universal quantifiers in $\pi$ and $y$ is the index of the sequence of ordinal numbers which correspond to existential quantifiers in $\pi . " \quad$ Thus if $t$ begins with four quantifiers of which the $0^{\text {th }}$ and $2^{\text {nd }}$ are universal and the $1^{\text {st }}$ and $3^{\text {rd }}$ are existential as in the particular example given above then $x$ is the index of the sequence $(0,2)$ and $y$ is the index of the sequence $(1,3)$. Then the following relation is closely connected with $A_{\psi} . \quad$ " $t$ is an $L$-sentence and $Q_{p r}(t, x, y)$ holds, and $z$ is the index of a Skolem operator $\psi$ from sequences with domain $\sigma_{1}$ to sequences with domain $\sigma_{2}$. Moreover, $u$ is the index of a particular finite partial sequence of $L$-individuals $\tau$, and $v$ is the $L$-sentence resulting from the substitution of the elements of $\tau$ for the variables corresponding to $\sigma_{1}$ and of the elements of $\psi(\tau)$ for the variables which correspond to $\sigma_{2}$, in the $L$-wff which results from the deletion of $\pi . " \quad$ We denote this relation by $B_{\psi}(t, x, y, z, u, v)$. Observe that in the present context we find it convenient to introduce the Skolem operator as an operation between sequences of $L$-symbols, and not of individuals of any 
particular model.

Let $G$ be any sentence of $L$-individuals. We shall presently make use of a relativized version of $B_{\psi}$, to be denoted by $B_{\psi G}(t, x, y, z, u, v)$ which is to hold if $B_{\psi}(t, x, y, z, u, v)$ holds and if moreover $u$ is the index of a sequence of $L$-individuals which belong to $G$, and $\psi$ (with index $z$ ) transforms all sequences with elements in $G$ into sequences with elements in $G$. Let $Q_{G}(x)$ be the relation which determines $G$ in $U$, and let $R_{G}(x, u)$ signify that the values of $\tau$ (determined by $u$ ) are in $G$ and the domain of $\tau$ is $\sigma_{1}$ (given by $x$ ).

Let $G_{T}$ be the set of $L$-sentences which are defined in a structure $M$ according to some correspondence between $L$-symbols and individuals, relations, and functors of $M$ as explained in the previous chapter. Suppose that $G$ is the set of $L$-individuals which correspond to individuals of $M$, determined by $Q_{G}(x)$, and let $G_{M}$ be the set of all $L$-sentences which hold in $M$, determined by $Q_{M}(x)$, as before. Then the following sentence holds in $U$

$$
\text { 5.1. } \begin{array}{r}
(\forall t)(\forall x)(\forall y)\left[Q _ { p r } ( t , x , y ) \supset \left[Q _ { u } ( t ) \equiv \left[( \exists z ) ( \forall u ) ( \forall v ) \left[\left[R_{G}(x, u) \wedge\right.\right.\right.\right.\right. \\
\left.\left.\left.\left.\left.B_{\psi G}(t, x, y, z, u, v)\right] \supset Q_{M}(v)\right]\right]\right]\right] .
\end{array}
$$

5.1 may be regarded as a formal statement of the fact that the truth of a sentence in a given model can be expressed in terms of the existence of appropriate Skolem functions as described in detail at the beginning of this section for the case of a sentence in prenex normal form. Passing to $U^{\prime}$, we see that 5.1 still expresses a certain connection between a structure $M^{\prime}$ in $U^{\prime}$ as introduced in section 3 and the set of $L^{\prime}$-sentences which are defined in $U^{\prime}$ according to given correspondences between $L^{\prime}$-symbols and individuals, relations, or functors of $M^{\prime}$. However, we have to bear in mind that the sets of sequences which occur in the definition of a Skolem operator in $U^{\prime}$ are confined to sequences of pseudo-finite character. On the other hand, the notion of substitution retains its meaning.

So far we have relied on the passage from $U$ to $U^{\prime}$ not only in the formulation of the non-standard language $L^{\prime}$ but also in its model - theoretic interpretation. We shall now introduce an alternative truth definition which relies directly on the notion of Skolem functions. It will be convenient to formulate this new concept only for a certain subset of the set of $L^{\prime}$-sentences whose elements have a relatively simple structure. While it would not be hard to 
widen the scope of our definition somewhat, it might be difficult to extend it to the set of all $L^{\prime}$-sentences.

For given $U$ and $U^{\prime}$, and a language $L^{\prime}$ in $U^{\prime}$, we define subsets of $L^{\prime}$-terms, $L^{\prime}$-atomic formulae, etc. as follows.

An $L^{\prime}$-term will be called simple if it represents the result of a finite number of applications of functors with a finite number of arguments. An $L^{\prime}$ atomic formula is simple if it consists of an $L^{\prime}$-relation with a finite number of arguments filled by simple $L^{\prime}$-terms. In this connection, the word finite is to be interpreted in the absolute sense so that simple terms and relations are constructed from their atomic symbols exactly as in the standard lower predicate calculus.

Simple $L^{\prime}$-wff are defined inductively as follows. An $L^{\prime}$-atomic formula is a simple $L^{\prime}$-wff. The negation of a simple $L^{\prime}$-wff is simple. An $L^{\prime}$-wff which is obtained from a set of simple $L^{\prime}$-wff by repeated conjunction alone is simple. An $L^{\prime}$-wff which is obtained from a set of simple $L^{\prime}$-wff by repeated disjunction alone is simple. Finally, an $L^{\prime}$-wff which is obtained from a simple $L^{\prime}$-wff by repeated quantification is simple. Note that the last three operations may involve an infinite number of conjunctions, or disjunctions, or quantifications, and that both universal and existential quantification may appear in the same operation of repeated quantification.

Every simple $L^{\prime}$-wff which is obtained by negation determines uniquely the $L^{\prime}$-wff from which it is obtained. Every simple $L^{\prime}$-wff which is obtained by conjunction determines uniquely a sequence of simple $L^{\prime}$-wff from which it is obtained by repeated conjunction and which are not themselves conjunctions. A corresponding fact holds for disjunctions. Every simple $L^{\prime}$-wff which is obtained by quantification determines uniquely a simple $L^{\prime}$-wff from which it is obtained by repeated quantification and which is not itself obtained by quantification. The truth of all these statements follows from the fact that they hold for the sentences of $L$ in $U$ and can be transferred to the sentences of $L^{\prime}$ in $U^{\prime}$.

The rank of a simple $L^{\prime}$-wff which is an atomic $L^{\prime}$-wff is, by definition, 0 . The rank of a simple $L^{\prime}$-wff which is obtained by negation from a simple $L^{\prime}$ wff of rank $n$ is, by definition, $n+1$. The rank of a simple $L^{\prime}$-wff which is obtained by repeated conjunction from the particular sequence of $L^{\prime}$-wff 
mentioned in the preceding sequence is, by definition of rank $n+1$ if all the elements of the sequence are of rank not exceeding $n$ and at least one of them is of rank $n$. A similar definition is introduced for disjunctions. Finally, if a simple $L^{\prime}$-wff is obtained by repeated quantification from a simple $L^{\prime}$-wff which is not itself obtained by quantification, and which is of rank $n$ then the rank of the quantified $L^{\prime}$-wff is again defined to be $n+1$. These definitions assign unique ranks to the elements of a subset $\Omega$ of the set of simple $L^{\prime}$-wff. The elements of $\Omega$ will be said to be simple $L^{\prime}$-wff of finite rank.

Now let $M$ be a structure in the standard sense of the lower predicate calculus, i.e. with finitary relations and functors (if any) but not necessarily contained in either $U$ or $U^{\prime}$. A one-to-one correspondence between the individuals, relations, and functors of $M$ and some $L^{\prime}$-individuals, $L^{\prime}$-relations, and $L^{\prime}$-functors (constituting sets $G^{\prime}, G_{R}^{\prime}, G_{F}^{\prime}$ ) then determines a set of elements of $\Omega$ which are defined in $M$, i.e. those simple $L^{\prime}$-atomic formulae whose $L^{\prime}$-individuals, etc., belong to the correspondence, and all other simple $L^{\prime}$-wff of finite rank that are constructed from them by a finite number of negations and of repeated conjunctlons, disjunctions, and quantifications. The set of these elements of $\Omega$ will be denoted by $\Omega_{W}$.

The external truth definition for sentences of $\Omega_{W}$ with respect to the structure $M$ (for the given correspondence of atomic symbols) will now be introduced in the following way.

For elements of $\Omega_{W}$ which are of rank 0 we use the standard truth definition. That is to say if a sentence of $\Omega_{W}$ which is of rank 0 is of the form $r\left(a_{1}, \ldots, a_{n}\right)$ where $r$ is an $L^{\prime}$-relation and $a_{1}, \ldots, a_{n}$ are $L^{\prime}$-individuals then $r\left(a_{1}, \ldots, a_{n}\right)$ is true (or holds) in $M$ externally if and only if the corresponding $n$-tuple of individuals of $M$ belongs to the relation of $M$ that corresponds to $r$. If the sentence is of the form $r\left(t_{1}, \ldots, t_{n}\right)$ where $t_{1}, \ldots, t_{n}$ are $L^{\prime}$-terms without variables then we first evaluate these terms, i.e. we replace them by $L^{\prime}$-individuals which correspond to the functional values of $t_{1}, \ldots, t_{n}$ in $M$ and then proceed as before.

Suppose now that we have already assigned external truth values to all sentences of $\Omega_{W}$ of rank $<n$ where $n$ is a positive integer. Let $a$ be any sentence of $\Omega_{w}$ which is of rank $n$. If $n$ is a conjunction then, as explained above, $a$ is obtained by repeated conjunction from a specific sequence of $\Omega_{W^{-}}$ 
sentences which are not conjunctions and which are of rank $\leq n-1$. If and only if all these are true in $M$ externally, then we define that $a$ is true in $M$ externally. Similarly, if $a$ is a disjunction then we define that $a$ is true externally if and only if at least one of the elements of the corresponding sequence is true externally. If $a$ is a negation, of an $\Omega_{W}$-sentence, $b$ say, then $a$ is true externally if and only if $b$ is not true externally.

Finally, suppose that $a$ is obtained by repeated quantification from an element of $\Omega_{W}$ which is not itself obtained by quantification, $b$ say, where $b$ is a predicate not a sentence. We may symbolize the connection between $a$ and $b$ briefly by $a=q b$ where $q$ indicates a finite or infinite sequence of quantifiers. The quantified variables in $q$ constitute an ordered set of length $n$, where $n$ is a finite or infinite natural number. The natural numbers whose places in $q$ are occupied by universal and existential quantifiers respectively (beginning with 0 ) constitute two disjoint sets of natural numbers in $M_{0}^{\prime}, \sigma_{1}$ and $\sigma_{2}$ say. Let $\Phi_{i}$ be the set of all partial pseudo-finite sequences of elements of $G^{\prime}$ with domain $\sigma_{i}, i=1,2$. Let $\Psi$ be the set of all Skolem operators from $\emptyset_{1}$ to $\emptyset_{2}$, where the underlying set $\sigma_{1} \cup \sigma_{2}$ is ordered as in $M_{0}^{\prime}$. Then we shall say that $a$ is true in $M$ externally if there exists a Skolem operator $\psi \in \Psi$ such that if we substitute the individuals of the set $\left(\phi_{1}, \psi\left(\phi_{1}\right)\right)$ for the corresponding free variables of $b$ then we obtain an $\Omega_{W}$-sentence (of rank $n-1$ ) which is true in $M$ externally. In this connection, we recall that the set of pseudo-finite sequences is defined by means of an index set $J^{\prime}$ in $U^{\prime}$. On the other hand, we have not restricted $\Psi$ by the stipulation that its elements be given by some index set. It might appear at first sight that this procedure is somewhat arbitrary. However, in actual fact we only introduce the restriction on the elements of $\Phi_{1}$ and $\Phi_{2}$ (i.e. to pseudo-finite sequences) in order to ensure that the result of substituting $\left(\phi_{1}, \phi\left(\phi_{1}\right)\right)$ for the free variables of $b$ yields an $L^{\prime}$ sentence at all. In fact, it can be shown without difficulty that this last condition is entirely equivalent to the above restriction on the elements of $\Phi_{1}$ and $\Phi_{2}$. We shall see presently that it implies no corresponding restriction on the elements of $\Psi$.

This completes our definition of external truth. It is not difficult to verify that if we substitute sentences of $\Omega_{W}$ for the propositional variables in any tautology of the standard propositional calculus then we obtain a sentence 
which holds in $M$ externally. Similarly if we substitute an element of $\Omega_{W}$ with a simple free variable for the predicate variable $F$ in one of the formulae $[(\forall x) F(x)] \supset F(a)$ or $F(a) \supset[(\exists x) F(x)]$ then we obtain a sentence of $\Omega_{w}$ which holds in $M$ externally (provided we obtain a wff at all as usual). Finally, it is not difficult to verify that the modus ponens and the two rules of deduction of the predicate calculus also lead from sentences of $\Omega_{W}$ which are true in $M$ externally to sentences of the same kind. Accordingly, we may say that the external truth definition is a reasonable one. We might further suspect that it actually coincides with the interior truth definition in cases where both definitions are applicable. Thus, let $M^{\prime}$ be a structure in $U^{\prime}$ which corresponds to some structure in $U$ and let $G_{T}$ be the set of $L^{\prime}$-sentences defined in $M$ according to some given correspondences between $L^{\prime}$-symbols and individuals, relations, and functions of $M^{\prime}$, as in section 3. By the same correspondences, the subset $G_{T}^{*}$ of $G_{T}$ which consists of the simple $L^{\prime}$-sentences of finite rank in $G_{T}$ is defined in $M^{\prime}$ according to the rules of the present section. It is not difficult to see that if a sentence of $G_{T}^{*}$ does not involve any quantifiers then it holds in $M^{\prime}$ according to the external truth definition if and only if it holds in $M^{\prime}$ according to the internal truth definition.

Indeed, for a simple $L^{\prime}$-sentence of rank $0, a$, the external truth definition is the same as for the standard predicate calculus and, since 4.5 and 4.6 hold also in $U^{\prime}$, the internal truth definition for $a$ (whose relations and functions are finitary) also is the same as for the standard predicate calculus. It follows that the two definitions coincide in this case.

Supposing that we have already proved our assertion for sentences of rank smaller than $n$, where $n>0$, let $a$ be a simple $L^{\prime}$-sentence of rank $n$ which does not involve any quantifiers. If $a$ is obtained by conjunction, then (see above) it is a repeated conjunction of a set of $L^{\prime}$-sentences of rank $<n$ which are not themselves conjunctions.

According to the external truth definition, $a$ is true in $M^{\prime}$ if and only if all the elements of the set of conjuncts just mentioned are true in $M^{\prime}$. Now this is a condition which holds in $U$ and can be formulated as a sentence of $K$ and, accordingly, holds also in $U^{\prime}$. It follows that the external and internal truth definitions for $a$ coincide. The argument is similar for disjunctions and rather simpler for negations. 
Now suppose that the $L^{\prime}$-sentence $a$ has been obtained from a simple $L^{\prime}$. sentence $b$ of finite rank by (possibly, infinitely repeated) quantification, where $b$ is free of quantifiers. Within $U$, if an $L$-sentence in prenex normal form holds in a structure $M$ then there exists a corresponding Skolem operator $\psi$. This fact can be formulated as a sentence of $K$ and, accordingly, holds also in $U^{\prime}$. We conclude that if $a$ holds in $M^{\prime}$ according to the internal truth definition, then it holds in $M^{\prime}$ also according to the external truth definition. The converse does not follow in this way since we have admitted Skolem operators in $U^{\prime}$ which are not necessarily indexed in $U^{\prime}$. More precisely, we shall show in the next section that the internal and external truth definitions do not coincide in all cases.

6. Discrepancy between internal and external truth definitions. Let $U$ be the structure considered throughout this paper and let $M_{0}^{*}$ be the selected standard model of the natural numbers and $L$ the standard Lower predicate calculus within $U$, as before. $K$ being again the set of all sentences which are defined and hold in $U$, let $H$ be the set of sentences of $K$ which do not include any individual constants. Let $W$ be any model of $H$. We claim that $W$ contains a partial structure, $W_{0}$, which is isomorphic to $U$.

Indeed, for any element $a$ of $U, K$ includes a relation $R_{a}(x)$ which holds only for $a$. Thus the sentences

$$
R_{a}(a)
$$

$$
(\exists x) R_{a}(x) \text {, }
$$

$$
(\forall x)(\forall y)\left[R_{a}(x) \wedge R_{a}(y) \supset I(x, y)\right]
$$

belong to $K$. 6.2 and 6.3 belong also to $H$ and, accordingly hold in $W$. It is easy to see that we may impose the condition that $I(x, y)$ is the relation of identity also in $W$ without limiting the validity of our argument. Let $W_{0}$ be the partial structure of $W$ which consists of all elements $b$ in $W$ for which there exists an $a$ in $U$ such that $R_{a}(b)$ holds in $W$. This establishes a one-toone correspondence $C: \mathrm{a} \leftrightarrow b$ between the elements of $U$ and the elements of $W_{0}$. We are going to show that $C$ establishes an isomorphism between $U$ and $W_{0}$.

Let $R\left(x_{1}, \ldots, x_{n}\right)$ be any relation in $U$ and let $a_{1}, \ldots, a_{n}$ be a set of 
elements of $U$. Then $R\left(a_{1}, \ldots, a_{n}\right)$ holds in $U$ if and only if the sentence

6.4 .

$$
\left(\forall x_{1}\right) \cdots\left(\forall x_{n}\right)\left[R_{a_{1}}\left(x_{1}\right) \wedge \cdots \wedge R_{a_{n}}\left(x_{n}\right) \supset R\left(x_{1}, \ldots, x_{n}\right)\right]
$$

belongs to $K$ and hence to $H$. Let $a_{i} \leftrightarrow b_{i}$ under the correspondence $C$ then $R_{a_{i}}\left(b_{i}\right)$ holds in $W$. Thus if $R\left(a_{1}, \ldots, a_{n}\right)$ holds in $U$, then 6.4 belongs to $H$ and so $R\left(b_{1}, \ldots, b_{n}\right)$ holds in $W_{0}$. Conversely, if $R\left(b_{1}, \ldots, b_{n}\right)$ holds in $W_{0}$ then 6.4 holds in $H$ and so $R\left(a_{1}, \ldots, a_{n}\right)$ holds in $U$. This shows that $C$ is an isomorphic correspondence with respect to the relations of the two structures and a similar argument applies with respect to the functions. It follows that if we identify the $b_{i}$ with the corresponding $a_{i}$ then we may regard $W$ as an extension of $U$, and a model of $K$.

Let $S_{0}^{*}(x, y, z)$ and $P_{0}^{*}(x, y, z)$ be two relations which determine addition and multiplication within the set of natural numbers in $U, M_{0}^{*}$. More precisely, we shall suppose that $S_{0}^{*}(a, b, c)$ holds if and only if $a, b, c$ belong to $M_{0}^{*}$ and $a+b=c$, and $P_{0}^{*}(a, b, c)$ holds if and only if $a, b, c$ belong to $M_{0}^{*}$ and $a b=c$. In particular, $S_{0}^{*}(a, b, c)$ and $P_{0}^{*}(a, b, c)$ do not hold if at least one of $a, b, c$ is outside $M_{0}$. Also, let $I_{0}^{*}(x, y)$ coincide with the relation of identity $I^{*}(x, y)$ on $M_{0}^{*}$, while $I_{0}^{*}(a, b)$ does not hold if at least one of the constants $a, b$ is outside $M_{0}^{*}$. Thus $I_{0}^{*}(a, a)$ holds only for element $a$ of $M_{0}^{*}$.

In section 2 above, we considered a model of the natural numbers, $M_{0}$, which included the relation of identity $I(x, y)$ and the functions $a(x, y), \pi(x, y)$ and $\phi(x)$, i.e. $x+y, x y$ and $x^{\prime}=x+1$. It is not difficult to show, and is usually taken for granted, that we may replace the three functions just mentioned by two three-place predicates, $S(x, y, z)$ and $P(x, y, z)$, which stand for $x+y=z$ and $x y=z$. Thus, every relation $Q\left(x_{1}, \ldots, x_{n}\right)$ within the domain of natural numbers which is definable in the Lower predicate calculus in terms of the relation of identity, $I(x, y)$ and the functions $\sigma, \pi$ and $\phi$, is definable also in terms of $I, S$ and $P$. This applies to the model of the natural numbers, $M_{0}$, as introduced in section 2, i.e. without reference to any structure $U$ which includes $M_{0}$. Suppose however, that $M_{0}$ coincides with $M_{0}^{*}$ as above. In that case the relations $I, S, P$ are obtained by restricting $I_{0}^{*}, S_{0}^{*}, P_{0}^{*}$ to $M_{0}^{*}$.

Let $Q\left(x_{1}, \ldots, x_{n}\right), n \geq 1$, be any relation which constitutes a subset of $M_{0}=M_{0}^{*}$ (i.e. which holds for the $n$-tuples of a certain set of natural numbers). Then we claim that $Q$ is definable in terms of $I, S$, and $P$ if and only if it is 
definable in terms of $I_{0}, P_{0}$ and $S_{0}$ (with reference to $U$ ).

To prove our assertion we denote by $R_{0}^{*}(x)$ the relation which defines $M_{0}^{*}$ within $U$. Thus, $R_{0}^{*}(a)$ holds in $U$ precisely when $a$ belongs to $M_{0}^{*}$. Let $Q\left(x_{1}, \ldots, x_{n}\right)$ be a predicate which is defined in $M_{0}=M_{0}^{*}$ in terms of the relations $I, S$, and $P$ (without reference to $U$ ), and let $Q\left(x_{1}, \ldots, x_{n}\right)$ be obtained from $Q$ by relativizing that predicate with respect to $R_{0}^{*}$ and by replacing $I, S, P$ everywhere by $I_{0}^{*}, S_{0}^{*}, P_{0}^{*}$, respectively. Let $a_{1}, \ldots, a_{n}$ be elements of $M_{0}$. Then $Q\left(a_{1}, \ldots, a_{n}\right)$ holds in $M_{0}$ if and only if $Q_{0}\left(a_{1}, \ldots\right.$, $\left.a_{n}\right)$ holds in $U$. It follows that the predicate $6.5 T\left(x_{1}, \ldots, x_{n}\right)=R\left(x_{1}\right) \wedge \cdots$ $\wedge R\left(x_{n}\right) \wedge Q^{*}\left(x_{1}, \ldots, x_{n}\right)$ determines the same set as $Q$. where $T$ is interpreted with reference to $U$. Now $(\forall x)\left[R_{0}^{*}(x) \equiv I_{0}^{*}(x, x)\right]$ holds in $U$. It follows that if $T^{*}\left(x_{1}, \ldots, x_{n}\right)$ is obtained from $T\left(x_{1}, \ldots, x_{\eta}\right)$ by substituting $I_{0}^{*}(x, x)$ everywhere for $R_{0}^{*}(x)$ then $T^{*}$ defines the same set as $T$. Thus $T^{*}$ is defined in terms of $I_{0}^{*}, S_{0}^{*}, P_{0}^{*}$ and determines the same set within $U$ as $Q$ determines in $M_{0}$, without reference to $U$.

To prove the converse, we introduce a relation of order $0, F^{*}$ ("False") which does not hold in $U$, by definition. Let $F$ be the restriction of $F^{*}$ to $M_{0}$. Thus, $F$ is defined but does not hold in $M_{0}$. Let $Q^{*}\left(x_{1}, \ldots, x_{n}\right), n \geq 1$, be a predicate which is formulated in terms of $I_{0}^{*}, S_{0}^{*}, P_{0}^{*}$ and which defines a subset of $M_{0}^{n}$ (the space of points $\left(a_{1}, \ldots, a_{n}\right)$ with $\left.a_{i} \in M_{0}\right)$ when $M_{0}=M_{0}^{*}$ is regarded as a partial structure of $U$. It will be sufficient to consider only $Q^{*}$ which are in prenex normal form,

6.6.

$$
Q^{*}\left(x_{1}, \ldots, x_{n}\right)=q_{1} q_{2} \cdots q_{k} Z\left(x_{1}, \ldots, x_{n}, y_{1}, \ldots, y_{k}\right)
$$

where $Z$ does not contain any quantifiers and where $q_{i}$ quantifies $y_{i}, i=1$, $\ldots, k$.

We define the predicates $Q_{0}\left(x_{1}, \ldots, x_{n}, y_{1}, \ldots, y_{k}\right)$, $Q_{1}\left(x_{1}, \ldots, x_{n}, y_{1}, \ldots, y_{k-1}\right), \ldots, Q_{k}\left(x_{1}, \ldots, x_{n}\right)$ as follows.

6.7. $Q_{0}\left(x_{1}, \ldots, x_{n}, y_{1}, \ldots, y_{k}\right)=Z\left(x_{1}, \ldots, x_{n}, y_{1}, \ldots, y_{k}\right)$.

Supposing that $Q_{j}\left(x_{1}, \ldots, x_{n}, y_{i}, \ldots, y_{k-j}\right)$ has been defined already, $0 \leq j<k$, we distinguish two cases.

If $q_{k-j}$ is an existential quantifier, $q_{k-j}=\left(\exists y_{k-j}\right)$ then we put 
6.8. $Q_{j+1}\left(x_{1}, \ldots, x_{n}, y_{1}, \ldots, y_{k-j-1}\right)=\left(\exists y_{k-j}\right)\left[R_{0}\left(y_{k-j}\right)\right.$

$$
\left.\wedge\left[Q_{j}\left(x_{1}, \ldots, x_{n}, y_{1}, \ldots, y_{k-j}\right) \wedge Q_{j}^{*}\left(x_{1}, \ldots, x_{n}, y_{1}, \ldots, y_{k-j-1}\right)\right]\right]
$$

where $Q_{j}^{*}$ is obtained from $Q_{j}$ by replacing every atomic formula in $Q_{j}$ which contains $y_{k-j}$ by $F_{0}^{*}$. If $q_{k-j}$ is a universal quantifier, $q_{k-j}=\left(\forall y_{k-j}\right)$, we put

$$
\begin{aligned}
& Q_{j+1}\left(x_{1}, \ldots, x_{n}, y_{1}, \ldots, y_{k-j-1}\right)=\left(\forall y_{k-j}\right)\left[R_{0}^{*}\left(y_{k-j}\right) \supset\right. \\
& {\left[Q_{j}\left(x_{1}, \ldots, x_{n}, y_{1}, \ldots, y_{k-j}\right)\right.}\left.\left.\wedge Q_{j}^{*}\left(x_{1}, \ldots, x_{n}, y_{1}, \ldots, y_{k-j-1}\right)\right]\right]
\end{aligned}
$$

where $Q_{j}^{*}$ is defined as before.

Taking the former case, we observe that the sentence

6. 10. $\left(\forall x_{1}\right) \cdots\left(\forall x_{n}\right) \cdots\left(\forall y_{k-j-1}\right)\left[\left(\exists y_{k-j}\right) Q_{j}\left(x_{1}, \ldots, x_{n}, y_{1}, \ldots, y_{k-j}\right) \equiv\right.$

$$
\left.Q_{j+1}\left(x_{1}, \ldots, x_{n}, y_{1}, \ldots, y_{k-j-1}\right)\right]
$$

holds in $U$. Indeed, the sentence

6.11. $\left(\forall x_{1}\right) \cdots\left(\forall x_{n}\right) \cdots\left(\forall y_{k-j-1}\right)\left[\left[\left(\exists y_{k-j}\right) Q_{j}\left(x_{1}, \ldots, x_{n}, y_{1}, \ldots, y_{k-j}\right) \equiv\right.\right.$

$$
\begin{array}{r}
{\left[( \exists y _ { k - j } ) [ [ R _ { 0 } ^ { * } ( y _ { k - j } ) \wedge Q _ { j } ( x _ { 1 } , \ldots , x _ { n } , y _ { 1 } , \ldots , y _ { k - j } ) ] ] \vee \left[\left(\exists y_{k-j}\right)\right.\right.} \\
\left.\left.\left[\sim R_{0}^{*}\left(y_{k-j}\right) \wedge Q_{j}\left(x_{1}, \ldots, x_{n}, y_{1}, \ldots, y_{k-j}\right)\right]\right]\right]
\end{array}
$$

is a theorem of the Lower predicate calculus. But

$$
\begin{array}{r}
\left(\forall x_{1}\right) \cdots\left(\forall x_{n}\right) \cdots\left(\forall y_{k-j}\right)\left[\left[\sim R_{0}^{*}\left(y_{k-j}\right) \wedge Q_{j}\left(x_{1}, \ldots, x_{n}, y_{1}, \ldots, y_{k-j}\right)\right] \equiv\right. \\
\left.Q_{j}^{*}\left(x_{1}, \ldots, x_{n}, y_{1}, \ldots, y_{k-j}\right)\right]
\end{array}
$$

holds in $U$ since any atomic sentence of $Q_{j}$ that contains an individual $a$ which is in $U$ but outside $M_{0}^{*}$, cannot hold in $U$. It follows, by the rules of the Lower predicate calculus that the sentences

$$
\begin{aligned}
\left(\forall x_{1}\right) \cdots\left(\forall x_{n}\right) \cdots\left(\forall y_{k-j-1}\right)\left(\exists y_{k-j}\right)\left[\left[\sim R_{0}^{*}\left(y_{k-j}\right) \wedge\right.\right. \\
\left.\left.\quad Q_{j}\left(x_{1}, \ldots, x_{n}, y_{1}, \ldots, y_{k-j}\right)\right] \equiv Q_{j}^{*}\left(x_{1}, \ldots, x_{n}, y_{1}, \ldots, y_{k-j-1}\right)\right]
\end{aligned}
$$

and

6.12. $\left(\forall x_{1}\right) \ldots\left(\forall x_{n}\right) \ldots\left(\forall y_{k-j-1}\right)\left[\left[\left(\exists y_{k-j}\right)\left[\sim R_{0}^{*}\left(y_{k-j}\right) \wedge\right.\right.\right.$

$$
\left.\left.\left.Q_{j}\left(x_{1}, \ldots, x_{n}, y_{1}, \ldots, y_{k-j}\right)\right]\right] \equiv Q_{j}^{*}\left(x_{1}, \ldots, x_{n}, y_{1}, \ldots, y_{k-j-1}\right)\right]
$$

also hold in $U$. Combining 6.12 with 6.11 , we obtain 6.10 .

Suppose now that $q_{k-j}$ is a universal quantifier, $g_{k-j}=\left(\forall y_{k-j}\right)$. In that case, similar considerations show that

6.13. $\left(\forall x_{1}\right) \cdots\left(\forall x_{n}\right) \cdots\left(\forall y_{k-j-1}\right)\left[\left[\left(\forall y_{k-j}\right) Q_{j}\left(x_{1}, \ldots, x_{n}, y_{1}, \ldots, y_{k-j}\right)\right]\right.$

$$
\left.\equiv Q_{j+1}\left(x_{1}, \ldots, x_{n}, y_{1}, \ldots, y_{k-j-1}\right)\right]
$$

holds in $U$. Thus, in either case, 
6. 14. $\left(\forall x_{1}\right) \cdots\left(\forall x_{n}\right) \cdots\left(\forall y_{k-j-1}\right)\left[\left[q_{k-j} \boldsymbol{Q}_{j}\left(x_{1}, \ldots, x_{n}, y_{1}, \ldots, y_{k-j}\right)\right]\right.$ $\left.\equiv Q_{j+1}\left(x_{1}, \ldots, x_{n}, y_{1}, \ldots, y_{k-j-1}\right)\right]$ holds in $U$. Applying 6.14 for $j=0,1$, $\ldots, k-1$ and taking into account 6.7 , we conclude that $\left(\forall x_{1}\right) \cdots\left(\forall x_{n}\right)$ $\left[q_{1}, \ldots, q_{k} Q_{0}\left(x_{1}, \ldots, x_{n}, y_{1}, \ldots, y_{k}\right) \equiv Q_{k}\left(x_{1}, \ldots, x_{n}\right)\right]$

i.e. 6. 15. $\left(\forall x_{1}\right) \cdots\left(\forall x_{n}\right)\left[Q^{*}\left(x_{1}, \ldots, x_{n}\right) \equiv Q_{k}\left(x_{1}, \ldots, x_{n}\right)\right]$ holds in $U$.

Next, we define another sequence of predicates, $T_{0}\left(x_{1}, \ldots, x_{n}, y_{1}, \ldots, y_{k}\right)$, $T_{1}\left(x_{1}, \ldots, x_{n}, y_{1}, \ldots, y_{k-1}\right), \ldots, T_{k}\left(x_{1}, \ldots, x_{n}\right)$ by

6. 16. $T_{0}\left(x_{1}, \ldots, x_{n}, y_{1}, \ldots, y_{k}\right)=Z\left(x_{1}, \ldots, x_{n}, y_{1}, \ldots, y_{k}\right)$ and by

6. 17. $T_{j+1}\left(x_{1}, \ldots, x_{n}, y_{1}, \ldots, y_{k-j-1}\right)=q_{k-j}\left[T_{j}\left(x_{1}, \ldots, x_{n}, y_{1}, \ldots, y_{k-j}\right) \wedge\right.$

$$
\left.T_{j}^{*}\left(x_{1}, \ldots, x_{n}, y_{1}, \ldots, y_{k-j-1}\right)\right]
$$

for $0 \leq j<k-1$, where $T_{j}^{*}$ is obtained from $T_{j}$ by replacing every atomic formula in $T_{j}$ which contains $y_{k-j}$ by $F_{0}^{*}$.

By considering successive $j, j=0,1, \ldots, k$, we see that $Q_{j}\left(x_{1}, \ldots, x_{n}\right.$, $\left.y_{1}, \ldots, y_{k-j-1}\right)$ is obtained by relativizing the predicate $T_{j}\left(x_{1}, \ldots, x_{n}, y_{1}, \ldots\right.$, $\left.y_{k-j-1}\right)$ with respect to $R_{0}^{*}(x)$. It follows that the predicate $T\left(x_{1}, \ldots, x_{n}\right)$ which is obtained from $T_{k}\left(x_{1}, \ldots, x_{n}\right)$ by replacing $I_{0}^{*}, S_{0}^{*}, P_{0}^{*}, F_{0}^{*}$ everywhere by $1, S, P, F$, respectively, holds in $M_{0}$ for any $a_{1}, \ldots, a_{n}$ in $M_{0}$ if and only if $Q_{k}\left(x_{1}, \ldots, x_{n}\right)$ holds in $U$, i.e. by 6.15 , if and only if $Q^{*}\left(a_{1}, \ldots, a_{n}\right)$ holds in $U . \quad T\left(x_{1}, \ldots, x_{n}\right)$ is not as yet the required predicate since it contains, in addition to $I, S, P$, the relation $F$. However, replacing $F$ everywhere in $T\left(x_{1}, \ldots, x_{n}\right)$ by $(\forall x)[I(x, x) \wedge \sim I(x, x)]$ we obtain a predicate $Q\left(x_{\mathrm{i}}, \ldots, x_{n}\right)$ which is obtained in terms of $I, S$, and $P$ and which holds in $M_{0}$ without reference to $U$ if and only if $Q^{*}\left(x_{1}, \ldots, x_{n}\right)$ holds in $U$. This completes the proof of our assertion.

With $U$ and $M_{0}=M_{0}^{*}$ as before, let $L$ be the Lower predicate calculus within $U$ as introduced in seetion 3. Suppose that a set $G$ of $L$-individuals is in one-to-one correspondence with the elements of $M_{0}$ and let $j, s$, and $p$ be $L$-relations which correspond to the relations $I(x, y), S(x, y, z)$ and $P(x, y, z)$ in $M_{0}$, respectively. Thus, in the notation of section 4 with $M=M_{0}, G_{R}=\{j, s, p\}$ while $G_{F^{r}}$ is empty. Putting it in a less formal way, we may say that $j, s$, and $p$, denote the respective relations in $M_{0}$. Let $G_{W}$ be the set of $L$-wff formulated in terms of these $L$-symbols and let $G_{r}$ be the set of $L$-sentences which belong to $G_{W}$. Let $G_{P}$ be the set of elements of $G_{T}$ which are in prenex normal form 
and such that the matrix (the quantifier-free part of the sentence) is in Boolean normal form as a conjunction of disjunctions of atomic sentences and of negations of such sentences. Let $G_{T}^{*}$ be the set of elements of $G_{T}$ which hold in $M_{0}$ and let $G_{P}^{*}$ be the corresponding subset of $G_{P}$.

We may map the wff of $G_{W}$ on a subset $S_{W}$ of $M_{0}=M_{0}^{*}$ by the familiar process of arithmetization. Then $S_{W}$ is recursive. Let $S_{T}, S_{P}, S_{T}^{*}, S_{P}^{*}$ be the sets of natural numbers which correspond to $G_{T}, G_{P}, G_{T}^{*}$, and $G_{P}^{*}$ respectively in this mapping. Then $S_{r}$ and $J_{p}$ also are recursive. Moreover, since there is a recursive procedure for transforming any given sentence in prenex normal form with a matrix in Boolean normal form, there exists a recusive numbertheoretic relation $K(x, y)$ such that the first argument determines the second argument - i.e. $(\forall x)(\forall y)(\forall z)[K(x, y) \wedge K(x, z) \supset I(y, z)]$ - and such that for every element $a$ of $S_{T}$ there exists an element $b$ of $S_{P}$ for which $K(a, b)$ holds. Moreover, $K(a, b)$ holds only if $a$ is in $S_{T}$ and $b$ is in $S_{P}$ and $a$ and $b$ are the Gödel numbers of equivalent sentences. Since $K(x, y)$ is recursive, it is definable in terms of the relations $I, S$, and $P$.

Let $V_{T}(x)$ and $V_{P}(x)$ be the relations which determine the sets $S_{T}^{*}$ and $S_{P}^{*}$ in $M_{0}$, without reference to $U$. Then

6. 18 .

$$
(\forall x)\left[V_{T}(x) \equiv(\exists y)\left[K(x, y) \wedge V_{P}(y)\right]\right]
$$

holds in $M_{0}$. By Tarski's theorem (2. 4 above), $V_{T}(x)$ cannot be expressed in terms of the operations of addition, multiplication and succession and as remarked earlier, this is equivalent to the fact that $V_{T}(x)$ cannot be expressed in terms of $I, S$ and $P$. On the other hand, $K(x, y)$ can be so expressed. Hence, by $6.18, V_{P}(x)$ cannot be expressed in terms of the relations $I, S$ and $P$. Combining this conclusion with the result of the previous argument we obtain

6.19. TheOREM. $S_{P}^{*}$, regarded as a subset of $U$, is not defined by any predicate which is formulated in terms of the relations $I_{0}^{*}(x, y), S_{0}^{*}(x, y, z), P_{0}^{*}(x, y, z)$ alone.

As before, let $H$ be the set of all sentences which are defined in terms of the relations of $U$ but without individual constants, and which hold in $U$. In particular, $H$ contains sentences which involve the relation $I_{0}^{*}(x, y), S_{0}^{*}(x, y, z)$, $P_{0}^{*}(x, y, z)$ and $V_{P}(x)$. Let $H^{\prime}$ be obtained from $H$ by replacing all relations 
of $H$ except $I_{0}^{*}, S_{0}^{*}, P_{0}^{*}$, by distinct new relations. In particular, let $V_{P}^{\prime}(x)$ be the relation which replaces $V_{P}(x)$. We are going to prove

6. 20. Theorem. The set $H=H \cup H^{\prime} \cup\left\{(\exists x)\left[\sim\left[V_{P}(x) \equiv V_{P}^{\prime}(x)\right]\right]\right\}$ is consistent.

Proof. Suppose on the contrary that $H^{*}$ is contradictory. Then

$$
H \cup H^{\prime} \vdash(\forall x)\left[V_{P}(x) \equiv V_{P}^{\prime}(x)\right] .
$$

In 6.21, we may replace $H$ and $H^{\prime}$ by finite subsets $H_{1}$ and $H_{2}$ of $H$ and $H^{\prime}$ respectively. Moreover - if necessary by adding a number of sentences - we may suppose that $H_{2}$ is obtained by replacing the relations of $H$, except $I_{0}^{*}, S_{0}^{*}, P_{0}^{*}$ by the new relations of $H^{\prime}$. Let $X$ be the conjunction of the sentences of $H_{1}$ then the conjunction of the sentences of $H_{2}, H^{\prime}$ say is obtained from $X$ in the same manner. Thus, $X \in H, X^{\prime} \in H^{\prime}$ and

$$
X \cap X^{\prime} \vdash(\forall x)\left[V_{P}(x) \equiv V_{P}^{\prime}(x)\right] .
$$

Theorem 2.1 now implies that there exists a predicate $Q(x)$ which is defined in terms of $I_{0}^{*}, S_{0}^{*}$ and $P_{0}^{*}$ alone, such that

$$
X \vdash(\forall x)\left[V_{P}(X) \equiv Q(x)\right] .
$$

But $X \in H$ holds in $U$ and so 6.23 entails that $V_{P}(x)$ is expressible in terms of $I_{0}^{*}, S_{0}^{*}, P_{0}^{*}$. This contradicts 6.19 and proves 6.20 .

Let $U^{*}$ be a model of $H^{*}$. $U^{*}$ contains a model of arithmetic, $\bar{M}_{0}$, which is defined in terms of $I_{0}^{*}, S_{0}^{*}, P_{0}^{*} . \quad \bar{M}_{0}$ cannot be the standard model since in that model $(\forall x)\left[V_{P}(x) \equiv V_{P}^{\prime}(x)\right]$. We denote by $U_{1}$ the structure which is obtained from $U^{*}$ by taking into account only the relations of $H$, and by $U_{1}^{\prime}$ the corresponding structure which takes into account only the relations of $H^{\prime}$. Then $U_{1}$ and $U_{1}^{\prime}$ are models of $H$ and $H^{\prime}$ respectively. They have in common the non-standard model of arithmetic, $\bar{M}_{n}$, which is a proper extension of the standard model of arithmetic $M_{0}$ in $U$. Thus the theory of sections $2-5$ applies to both $U_{1}$ and $U_{1}^{\prime}$. The sets which constitute the Lower predicate calculus within $U_{1}$ and $U_{1}^{\prime}$, respectively, need not coincide. However, if $G_{1 W}$ and $G_{1 W}^{\prime}$ are the sets which correspond to $G_{W}$ in $U_{1}$ and $U_{1}^{\prime}$ respectively then there is a natural one-to-one correspondence between $G_{1 W}$ and $G_{1 W}^{\prime}$ in the Gödel numbering of their elements. That is to say if $a \in G_{1 W}$ and $a^{\prime} \in G_{1 W}^{\prime}$ then we define $a \leftrightarrow a^{\prime}$ if $a$ and $a^{\prime}$ have the same Gödel numbers in $\bar{M}_{0}$. This cor- 
respondence is an isomorphism in the sense that if $a \leftrightarrow a^{\prime}$ then $a$ and $a^{\prime}$ possess the same length $l$ (where $l$ is an element of $\bar{M}_{0}$ ). Moreover, the $n^{\text {th }}$ atomic symbols of $a$ and $a^{\prime}$ either are the "same" logical constants other than variables, or variables with the same Gödel numbers, or they denote the same extralogical constants (relations, natural numbers). The sentences of $G_{1 W}$ are mapped on the sentences of $G_{1 W}^{\prime}$ under this correspondence, $G_{1 T} \leftrightarrow G_{1 T}^{\prime}$, and the same applies to the sentences in prenex normal form, $G_{1 P} \leftrightarrow G_{1 P}^{\prime}$.

Now let $G_{1 P}^{*}$ and $G_{1 P}^{* \prime}$ be the sets of sentences in prenex normal form within the Lower predicate calculi of $U$ and $U^{\prime}$, respectively which hold in $\bar{M}_{0}$. Let $A$ and $A^{\prime}$ be the sets of Gödel numbers of $G_{1 P}^{*}$ and $G_{1 P}^{* \prime}$, then $A$ and $A^{\prime}$ consist of the elements of $\bar{M}_{0}$ which satisfy $V_{P}(x)$ and $V_{P}^{\prime}(x)$, respectively. $U^{*}$ is a model of $H^{*}$ and so the sentence $(\exists x)\left[\sim\left[V_{P}(x) \equiv V_{P}^{\prime}(x)\right]\right]$ holds in $U^{*}$. Thus, there exists an element $m$ of $U^{*}$ such that $\sim\left[V_{P}(m) \equiv V_{P}^{\prime}(m)\right]$ holds in $U^{*}$. It follows that either $V_{P}(m)$ holds in $U^{*}$ and $V_{P}^{\prime}(m)$ does not hold in $U^{*}$ or $V_{P}$ does not hold in $U^{*}$ and $V_{P}^{\prime}(m)$ holds in $U^{*}$. Clearly, it does not restrict the generality of our considerations to suppose that the former case applies. $m$ is the Gödel number of sentences $a$ and $a^{\prime}$ within the Lower predicate calculi of $U_{1}$ and $U_{1}^{\prime}$ respectively. Then $a \leftrightarrow a^{\prime}$, and so $a$ and $a^{\prime}$ are "isomorphic" in the sense detailed above. The sentence $a$ holds in $\bar{M}_{0}$, since $V_{P}(m)$ holds in $U^{*}$. $a$ belongs to $G_{1 P}$, which includes $G_{1 P}^{*}$ as a subset and so $a^{\prime}$ belongs to $G_{1 P}^{\prime}$. But $a^{\prime}$ does not belong to $G_{1 P}^{* \prime}$ since $V_{P}^{\prime}(m)$ does not hold in $U^{*}$, and so $a^{\prime}$ does not belong to $G_{1 T}^{* \prime}$ either.

Let $b$ and $b^{\prime}$ be the negations of $a$ and $a^{\prime}$ within the Lower predicate calculi of $U$ and $U^{\prime}$, respectively, then $b \leftrightarrow b^{\prime}$. Let $c$ be obtained from $b$ by the standard transformation of the negation of a sentence in prenex normal form into a sentence in prenex normal form, i.e. by changing the type of the quantifier of $b$, existential to universal and vice versa, while the sign of negation is shifted to the front of the matrix of $b$. We shall say, briefly, that $c$ is obtained from $a$ by negation and normalization. Thus, if $c^{\prime}$ is similarly obtained from $a^{\prime}$ by negation and normalization then $c \leftrightarrow c^{\prime}$.

$a$ holds in $\bar{M}_{0}$ and so $b$ and $c$ are defined but do not hold in $\bar{M}_{0}$, all according to the internal truth definition. On the other hand, $a^{\prime}$ is defined but does not hold in $\bar{M}_{0}$ and so $b^{\prime}$ and $c^{\prime}$ hold in $\bar{M}_{0}$, again according to the internal truth definition. 
Now all sentences in prenex normal form with matrices in Boolean normal form are simple formulae in the sense introduced in section 5 , of ranks not exceeding 4. Accordingly, we may apply the external truth definition to the sentences of $G_{1 P}$ and $G_{1 P}^{\prime}$. Since corresponding sentences of $G_{1 P}$ and $G_{1 P}^{\prime}$ are given by the same Gödel numbers and are isomorphic in the sense explained above, it will be seen that corresponding elements of $G_{1 F}$ and $G_{1 . F}^{\prime}$ either both hold or both do not hold in $\bar{M}_{0}$. But $a$ holds in $\bar{M}_{0}$ according to the external truth definition. Similarly, $c^{\prime}$ holds in $\bar{M}_{0}$ according to the internal truth definition and so both $c$ and $c^{\prime}$ hold in $\bar{M}_{0}$ according to the external truth definition. $b$ and $b^{\prime}$ are the negations of $a$ and $c^{\prime}$ respectively, and so these sentences do not hold in $\bar{M}_{0}$ according to the external truth definition either.

Disregarding the relations which were introduced on passing from $H$ to $H^{\prime}$ and recalling that our structure $U^{*}$ is also a model of $K$, we thus obtain the following theorem.

6.24. Theorem. There exists a model $U^{*}$ of $K$ including a model of arithmetic $\bar{M}_{0}$, and three sentences $a, b, c$, in the Lower predicate calculus $L^{*}$ within $U^{*}$ such that the following conditions are satisfied. $a$ is an $L^{*}$-sentence in prenex normal form with a matrix in Boolean normal form; $b$ is obtained from $a$ by negation and $c$ is obtcined from $b$ by normalization; accordingly, $c$ is again in prenex normal form. The sentence a holds in $\bar{M}_{0}$ according to the internal truth definition, and the sentences $b$ and $c$ do not hold in $\bar{M}_{0}$ according to that definition. The sentences $a$ and $c$ hold in $\bar{M}_{0}$ according to the external truth definition and the sentence $b$ does not hold in $\bar{M}_{0}$ accoraing to that definition.

Our argument was based on the assumption that $V(a)$ holds in $U^{*}$ while $V^{\prime}(a)$ does not hold in that structure. If the opposite is true we only have to interchange the roles of $U_{1}$ and $U_{1}^{\prime}$.

Theorem 6.24 shows that a particular sentence (i.e. $c$ ) may well be true in $\bar{M}_{0}$ according to the external truth definition but false according to the internal truth definition. Moreover, whereas our intuition which is derived from the finite case, would indicate that $b$ and $c$ (which is obtained from $b$ by normalization) must be true simultaneously, the opposite applies if we adopt the external truth definition. As mentioned in the introduction, an analogus 
phenomenon was noticed by $\mathrm{H}$. Steinhaus and J. Micielski for certain sequences of quantifiers of order type $\omega$. No such sequences are possible in our calculus and it is not clear whether the connection between the two phenomena is more than superficial.

We may make our result somewhat more precise by imposing certain conditions on the form of the prefix in $a$. Thus, every sentence in prenex normal form is equivalent to another sentence in prenex normal form in which the quantifiers alternate. For example in ordinary notation, $(\forall x)(\forall y)(\exists z)(\exists w)$ $Q(x, y, z, w)$, where $Q$ is free of quantifiers, is equivalent to $(\forall x)(\exists u)(\forall y)(\exists z)$ $(\forall v)(\exists u)[Q(x, y, z, w) \wedge I(u, u) \wedge I(v, v)]$ where $I(u, u)$ and $I(v, v)$ need be included only if we wish to avoid empty quantification. Accordingly, we may restrict the sets $G_{P}, G_{P}^{*}$ which were employed in the proof of 6.24. to sentences in whose prefix the quantifier alternate. This leads to conclusion that the sentences $a$ and $c$ in 6.24 may also be supposed to be of this type.

In the external truth definition we combine laws of formation which are based on non-standard arithmetic with a standard semantical approach. As a result we may employ a non-standard language $L^{\prime}$ even in order to describe the standard system of natural numbers, $M_{0}$. This contrasts with previous efforts to penetrate into the world of non-standard arithmetic by employing the standard Lower predicate calculus in order to discuss a non-standard model of arithmetic.

\section{REFERENCES}

[1] W. Craig, Three uses of the Herbrand-Gentzen theorem in relating model theory and proof theory, Journal of Symbolic Logic, vol. 22 (1957), pp. 269-285.

[2] L. Henkin. Completeness in the theory of types, Journal of Symbolic Logic, vol. 15 (1950), pp. 81-91.

[3] L. Henkin, Some remarks on infinitely long formulas, Infinitistic Methods-Proceedings of the Symposium on Foundations of Mathematics, Warsaw 1959-pub. 1961, pp. 167-183.

[4] A. Robinson, Model theory and non-standard arithmetic, Infinitistic Methods-Proceedings of the Symposium on Foundations of Mathematics, Warsaw 1959-pub. 1961, pp. 265-302.

[5] D. Scott and A. Tarski, The sentential calculus with infinitely long expressions, Colloquium Mathematicum, vol. 6 (1958), pp. 165-170.

[6] A. Tarski, Remarks on predicate logic with infinitely long expressions, Colloquium Mathematicum, vol. 6 (1958), pp. 171-176.

[7] A. Tarski, A. Mostowski and R. M. Robinson, Undecidable Theories, Studies in Logic and the Foundations of Mathemctics, Amsterdam 1953, 